\title{
ON AUTOMORPHISMS OF MARKOV CHAINS
}

\author{
WOLFGANG KRIEGER, BRIAN MARCUS AND SELIM TUNCEL
}

\begin{abstract}
We prove several theorems about automorphisms of Markov chains, using the weight-per-symbol polytope.
\end{abstract}

\section{INTRODUCTION}

The main purpose of this paper is to show how the weight-per-symbol polytope $(\overline{\mathrm{WPS}})$, introduced in [MT1, $\S 3]$ can be used in the study of automorphisms (i.e., measure-preserving conjugacies) of Markov chains, in particular, with regard to the question of which permutations of finitely many periodic cycles can be realized by such automorphisms. In a Markov chain, the weight of a cycle is the product of the transition probabilities around the cycle. The weight-per-symbol (wps) of a cycle is its weight normalized by its length. We study the periods of the set of cycles with a given wps-in particular, with reference to how the given wps sits in WPS. In $\S 1$, we establish the notion of a period for any wps which lies in the interior of $\overline{\text { WPS }}$, and we compute this period. (The growth rate of the number of cycles with a given wps and length is computed in [MT2].) We also show that any given path is a subpath of some cycle with prescribed large least period and prescribed wps, provided the wps lies in the interior of WPS. The latter is used heavily later in the paper. While the results of $\S 1$ are also contained implicitly in [MT2], our proof here is a bit different and more direct.

In [MT1, $§ 3$, a scaffold of Markov chains was associated to any given Markov chain: the scaffold consists of induced Markov chains, corresponding to the faces of WPS. In $\S 2$, we refine the scaffold by introducing boundary components, and using this we examine the set of (large) least periods of cycles with given wps in the boundary of WPS.

In $\S 3$, we look at the permutation of boundary components induced by an automorphism of a Markov chain. We give a necessary condition in terms of shift equivalence for the existence of an automorphism that maps one boundary component to another. This gives a necessary and sufficient condition, though hard to check in general, for the existence of an "eventual" automorphism that realizes a given permutation of boundary components. We also explore inert automorphisms on Markov chains, introduced by Wagoner [Wa], and show that these automorphisms must fix each boundary component. From this, we see

Received by the editors April 2, 1990.

1980 Mathematics Subject Classification (1985 Revision). Primary 28D20, 54H20, 58F20. 
that the permutation induced by an automorphism, $\varphi$, on the set of boundary components depends only on the automorphism of the dimension module induced by $\varphi$.

In $\S 4$, we apply the results of the previous sections to obtain conditions for switching cycles, of large least period, by an automorphism. In particular, we show that, given any wps in the interior of WPS, two cycles in different orbits with the same (large) least period and the given wps can be switched by an automorphism. In general, for wps in the boundary of WPS, the boundary components pose serious obstructions to switching cycles. However, if for each face of WPS, the scaffold gives a single mixing Markov chain, then the boundary components pose no obstruction, and the largeness requirement above on the size of least period, needed to switch cycles, is uniform over WPS. This generalizes a result of Boyle, Lind and Rudolph [BLR, Theorem 7.2].

Finally, in $\S 5$, we give necessary and sufficient conditions for the existence of a composition of measure-preserving involutions that realizes a given permutation of finitely many cycles of a Bernoulli shift. This generalizes a result of Boyle and Krieger [BK, 3.10].

We thank Valerio de Angelis for comments on an earlier draft of this paper, Mike Boyle for discussions of Appendix B, and Jack Wagoner for discussions of his paper [Wa2].

\section{BACKGROUND}

Let exp denote the group of exponential functions $t \mapsto a^{t}, a>0$. Then $Z^{+}$[exp] is the semiring of positive integral combinations of exponential functions. As in Parry-Tuncel [PT1], we define Markov chains via square matrices $A$ over $Z^{+}$[exp]. We let $A_{t}$ be the matrix $A$ evaluated at $t$. The directed graph $G(A)$ is the graph whose adjacency matrix is $A_{0}$. Let $E(A), S(A)$ denote the sets of edges, states of $G(A)$. For an edge $e$, let $\underline{i}(e), \underline{t}(e)$ denote its initial, terminal states. Let $E_{I J}(A)$ be the set of all edges $e$ with $\underline{i}(e)=I$ and $\underline{t}(e)=J$. Fixing a correspondence between $E_{I J}(A)$ and the set of exponential functions, with multiplicity, which occur in $A_{I J}$, we write $\mathrm{wt}_{A}(e)=a$ where $a^{t}$ is the exponential corresponding to $e$. Note that $G(A)$ and the function $\mathrm{wt}_{A}$ completely determine $A$, and most of the time this is the way we view $A$. However, the $Z^{+}$[exp]-matrix form is a useful notation.

We say that $A$ is irreducible if the matrix $A_{0}$ is. In this case, using the Perron-Frobenius Theorem [Se] we can transform $\mathrm{wt}_{A}$ so that it defines honest transition probabilities: for an edge $e$ from state $I$ to state $J$

$$
p(e)=\frac{\mathrm{wt}_{A}(e) r_{J}}{\lambda\left(A_{1}\right) r_{I}}
$$

where $\lambda\left(A_{1}\right)$ is the spectral radius of $A_{1}$, and $r=\left(r_{I}\right)$ is a corresponding right positive eigenvector. Let $P$ be the $Z^{+}[\exp ]-$ matrix defined by

$$
P_{I J}=\sum_{e \in E_{I J}(A)} p(e)^{t}
$$

and let $\pi$ be the unique stationary probability vector of the stochastic matrix $P_{1}$. Then, $A$ defines a Markov chain

$$
\Sigma_{A}=\left(\Sigma_{A_{0}}, \sigma_{A_{0}}, \mu_{A}\right)
$$


where

$$
\Sigma_{A_{0}}=\left\{x=\left(\cdots e_{-1} e_{0} e_{1} \cdots\right) \in E(A)^{Z}: \underline{t}\left(e_{i}\right)=\underline{i}\left(e_{i+1}\right)\right\},
$$

$\sigma_{A_{0}}: \Sigma_{A_{0}} \rightarrow \Sigma_{A_{0}}$ is the left shift map and $\mu_{A}$ is the measure defined by

$$
\mu_{A}\left(e_{1} \cdots e_{n}\right)=\pi_{\underline{i}\left(e_{1}\right)} p\left(e_{1}\right) \cdots p\left(e_{n}\right) .
$$

$\Sigma_{A_{0}}$ is the shift of finite type (SFT) defined by $A_{0}$.

As noted in [MT1, 1.3(2)], two $Z^{+}$[exp]-matrices $A, A^{\prime}$ define the same Markov chain iff $A^{\prime}=D^{-1} A D / c^{t}$, for some $c^{t} \in \exp$ and diagonal matrix $D$ over exp. Note that $P$ and $A$ above define the same Markov chain. $P$ is the unique stochastic form of the Markov chain defined by $A$, i.e., the unique $Z^{+}$[exp]-matrix $P$, with $P_{1}$ stochastic, that defines the same Markov chain as $A$.

Sometimes we consider $Z^{+}$[exp]-matrices $A$ which are merely nonwandering (i.e., whenever there is a path from state $I$ to state $J$ in $G(A)$, there is a path from state $J$ to state $I$ ). Then, $A$ defines a finite disjoint union of Markov chains, also called $\Sigma_{A}$, but the relative measures of these Markov chains is left undefined and is irrelevant for our purposes. We are naturally led to nonwandering matrices from irreducible matrices via the scaffold [MT1, §3]. However, except in explicitly stated places, $A$ is assumed irreducible.

For a path $\gamma, \underline{i}(\gamma), \underline{t}(\gamma)$ denote the initial, terminal states. A cycle is a path $\gamma$ such that $\underline{i}(\gamma)=\underline{t}(\gamma)$. For a cycle $\gamma=e_{1} \cdots e_{n}, \sigma(\gamma)=e_{2} \cdots e_{n} e_{1}$. The orbit of $\gamma$ is $\left\{\sigma^{i}(\gamma)\right\}$. The least period of $\gamma$ is the smallest $i>0$ such that $\sigma^{i}(\gamma)=\gamma$. The length $l(\gamma)$ of $\gamma$ is the number $n$ of edges that it traverses. The weight of a path $\gamma$ is $\mathrm{wt}(\gamma)=\mathrm{wt}_{A}(\gamma)=\prod_{i=1}^{n} \mathrm{wt}_{A}\left(e_{i}\right)$. If $\underline{t}(\gamma)=\underline{i}(\eta)$, then $\gamma \eta$ denotes the concatenation of $\gamma$ and $\eta$. And if $\gamma$ is a cycle, then $\gamma^{N}$ denotes the concatenation of $N$ copies of $\gamma$.

For a cycle $\gamma$, the weight-per-symbol of $\gamma$ is defined:

$$
\operatorname{wps}(\gamma)=\operatorname{wps}_{A}(\gamma)=\frac{\log \mathrm{wt}_{A}(\gamma)}{l(\gamma)} .
$$

$\mathrm{WPS}_{A}$ denotes the set of weights-per-symbol of all cycles $\gamma$ in $G(A)$; $\left\langle\mathrm{WPS}_{A}\right\rangle_{Q}$ is the rational vector space generated by $\mathrm{WPS}_{A}, \overline{\mathrm{WPS}}_{A}$ is the convex hull of $\mathrm{WPS}_{A}$ in $\left\langle\mathrm{WPS}_{A}\right\rangle_{Q}, \mathrm{EXT}_{A}$ is the set of extreme points of $\mathrm{WPS}_{A}$.

It was shown in [MT1, 3.2] that $\left\langle\mathrm{WPS}_{A}\right\rangle_{Q}$ is finite dimensional, $\overline{\mathrm{WPS}}_{A}$ is a polytope (i.e., $\mathrm{EXT}_{A}$ is finite), and each extreme point is the weight-per-symbol of a simple cycle. The relative interior, relative boundary of $\overline{\mathrm{WPS}}_{A}$ are denoted int $\overline{\mathrm{WPS}}_{A}, \partial \overline{\mathrm{WPS}}_{A}$ (here "relative" means relative to the affine hull of $\overline{\mathrm{WPS}}_{A}$. )

The WPS polytope is well-defined independent of the defining matrix in the sense that if $A$ and $A^{\prime}$ define the same Markov chain, then there is an affine isomorphism (over $Q$ ) : $\overline{\mathrm{WPS}}_{A} \rightarrow \overline{\mathrm{WPS}}_{A^{\prime}}$, which carries $\mathrm{WPS}_{A}$ to $\mathrm{WPS}_{A^{\prime}}$. (See [MT1, 3.3].) Since this isomorphism is affine, it must carry the facial structure of $\overline{\mathrm{WPS}}_{A}$ to that of $\overline{\mathrm{WPS}}_{A^{\prime}}$.

The ratio group, originally defined in $[\mathrm{Kr}]$, is defined by

$$
\Delta_{A}=\left\{\frac{\mathrm{wt}(\gamma)}{\mathrm{wt}\left(\gamma^{\prime}\right)}: \gamma, \gamma^{\prime} \text { are cycles in } G(A) \text { and } l(\gamma)=l\left(\gamma^{\prime}\right)\right\} .
$$

This group is free abelian and finitely generated. If $A$ and $A^{\prime}$ define the same Markov chain, then $\Delta_{A}=\Delta_{A^{\prime}}$. Parry and Schmidt [PS] showed that any 
$A$ can be replaced by an $A^{\prime}$ (in the sense that $A$ and $A^{\prime}$ define the same Markov chain) such that $\mathrm{wt}_{A^{\prime}}$ takes values in $\Delta_{A^{\prime}}=\Delta_{A}$. Such a matrix $A^{\prime}$ is called a $\Delta$-form. This form has several advantages (see [MT1]).

Since $\left\langle\mathrm{WPS}_{A}\right\rangle_{Q}$ is a finite dimensional vector space over $A,\left\langle\mathrm{WPS}_{A}\right\rangle_{Q}$ is isomorphic over $Q$ to $Q^{n}$, some $n$. In this way $\Delta_{A}$ and $\log \Delta_{A}$ can be identified with the integer lattice, $Z^{n}$. This identification makes these objects easier to visualize. We endow $\left\langle\mathrm{WPS}_{A}\right\rangle_{Q}$ with the sup norm, \|\| , inherited from identification with $Q^{n}$. This gives a well-defined topology on $\left\langle\mathrm{WPS}_{A}\right\rangle_{Q}$.

Finally, we list some of the other notation used in this paper.

$$
\mathscr{C}=\mathscr{C}(A)=\{\text { cycles } \gamma \text { with least period }=l(\gamma)\} .
$$

For $w \in \overline{\mathbf{W P S}}_{A}$, let

$$
\mathscr{C}_{w}=\mathscr{C}_{w}(A)=\{\gamma \in \mathscr{C}: \operatorname{wps}(\gamma)=w\}
$$

For a path $\alpha$ in $G(A)$, let

$$
\mathscr{C}_{w, \alpha}=\mathscr{C}_{w, \alpha}(A)=\left\{\gamma \in \mathscr{C}_{w}: \alpha \text { is a suffix of } \gamma\right\} .
$$

For a set $\mathscr{U}$ of cycles, let $\mathscr{L}(\mathscr{U})=\{l(\gamma): \gamma \in \mathscr{U}\}$.

Let $d_{A}$ denote the period of $A$ (i.e., the gcd of cycle lengths of $\left.\left.G(A)\right)\right)$. If $A$ is a $\Delta$-form, then for $w \in$ int $\mathrm{WPS}_{A}$, let

$$
d(w)=d_{A}(w)=\operatorname{lcm}\left(d_{A}, \operatorname{gcd}\left\{d \in \mathbf{N}: d w \in \log \Delta_{A}\right\}\right) .
$$

In general, we define $d_{A}(w)=d_{\bar{A}}(\bar{w})$ where $\bar{A}$ is a $\Delta$-form for $A$ and $\bar{w}$ is the image of $w$ via the affine correspondence $\mathrm{WPS}_{A} \rightarrow \mathrm{WPS}_{\bar{A}}$.

(This does not depend on the particular $\Delta$-form.)

\section{LONG CYCLES AND THE PERIOD OF A WEIGHT-PER-SYMBOL}

Theorem (1.1) below shows, among other things, that the set of least periods of cycles with a given wps, $w \in$ int $\mathrm{WPS}_{A}$, is eventually periodic with some easily computed period. In particular,

$$
\text { int } \mathrm{WPS}_{A} \subset \mathrm{WPS}_{A} \text {. }
$$

So, WPS ${ }_{A}$ can differ from $\overline{\mathrm{WPS}}_{A}$ only on $\partial \overline{\mathrm{WPS}}_{A}$. See (2.2) of this paper and [MT1, 3.5(5)] for some examples.

(1.1) Theorem. (1) For $w \in \operatorname{int} \overline{\mathrm{WPS}}_{A}, \mathscr{L}\left(\mathscr{C}_{w}\right) \subset d(w) \mathbf{Z}$.

(2) Assume that $G(A)$ does not consist entirely of a single cycle. Let $W$ be a closed subset of int $\overline{\mathrm{WPS}}_{A}$. Let $\alpha$ be a path in $G(A)$. Let $M \in N$. Then, $\exists N=N_{A}(\alpha, W, M)$ such that for $w \in W$

$$
\mathscr{L}\left(\mathscr{C}_{w}\right) \cap[N, \infty)=\mathscr{L}\left(\mathscr{C}_{w, \alpha}\right) \cap[N, \infty)=(d(w) Z) \cap[N, \infty)
$$

and moreover if $n \in \mathscr{L}\left(\mathscr{C}_{w}\right) \cap[N, \infty)$, there are at least $M$ cycles in $\mathscr{C}_{w, \alpha}$ in pairwise distinct orbits with length $=n$.

An immediate consequence is:

(1.2) Corollary. (1) int $\overline{\mathrm{WPS}}_{A} \subset \mathrm{WPS}_{A}$.

(2) For $w \in$ int $\overline{\mathrm{WPS}}_{A}, d(w)=\operatorname{gcd}\left(\mathscr{L}\left(\mathscr{C}_{w}\right)\right)$.

A much stronger statement than the "moreover" is contained in [MT2, Theorem 14]. For the proof of (1.1), we will need the following lemmas. 
(1.3) Lemma. Let $\gamma_{1}, \gamma_{2}, \ldots, \gamma_{k} \in \mathscr{C}$ be cycles in distinct orbits, and $k \geq$ 2. Let $\eta=\eta_{1} \eta_{2} \ldots \eta_{k}$ be a decomposition of a cycle $\eta$ into subpaths (some of which may be empty) such that $\gamma_{1} \eta_{1} \gamma_{2} \eta_{2} \cdots \gamma_{k} \eta_{k}$ is an (allowed) cycle in $G(A)$. Then, for sufficiently large positive integers $m_{1}, \ldots, m_{k}$, the cycle $\gamma \equiv$ $\gamma_{1}^{m_{1}} \eta_{1} \gamma_{2}^{m_{2}} \eta_{2} \cdots \gamma_{k}^{m_{k}} \eta_{k} \in \mathscr{C}$ (i.e., the least period of $\gamma$ is $l(\gamma)$ ).

Proof. Let $l_{i}=l\left(\gamma_{i}\right), l_{i}^{\prime}=l\left(\eta_{i}\right)$.

We use the following fact:

$$
\text { if } \gamma_{i}^{l_{j}} \text { is a subpath of } \gamma_{j}^{N} \text { for some } N \text {, then } i=j \text {. }
$$

To see this, observe that since the length of $\gamma_{i}^{l_{j}}$ is a multiple of $l_{j}$, if $\gamma_{i}^{l_{j}}$ is a subpath of $\gamma_{j}^{N}$, then $\gamma_{i}^{l_{j}}=\left(\sigma^{a}\left(\gamma_{j}\right)\right)^{b}$, for some $a, b$; but since $\gamma_{i}, \gamma_{j} \in \mathscr{C}$ and are in distinct orbits, we get $i=j$.

Let $m_{1}, m_{2}$ be large enough that

$$
\begin{gathered}
m_{2} l_{2}>l_{1} l_{2}+l_{1}, \\
m_{1} l_{1}>l_{1} l_{j}+l_{j}^{\prime}+l_{1} l_{j+1}, \quad j=2, \ldots, k-1, \\
m_{1} l_{1}>2\left(l_{1} l_{k}+l_{k}^{\prime}\right) .
\end{gathered}
$$

Let $\gamma$ be as in the lemma. Suppose $\sigma^{i}(\gamma)=\gamma$ for some $1 \leq i<l(\gamma)$. Then

$$
\sigma^{i}(\gamma)=\gamma \quad \text { for some } 1 \leq i \leq l(\gamma) / 2 .
$$

If $i \leq m_{1} l_{1}-l_{1}$, then since $\gamma_{1} \in \mathscr{C}, \gamma=\gamma_{1}^{\left\lfloor l(\gamma) / l_{1}\right\rfloor} \delta$ where $l(\delta)<l_{1}$. Then by (1) above, $\gamma_{1}^{l_{2}}$ is a subpath of $\gamma_{2}^{m_{2}}$, contrary to (0) above.

If $m_{1} l_{1}-l_{1}<i<l(\gamma)-\left(m_{k} l_{k}+l_{k}^{\prime}\right)$, then by (2) above, for some $j: 2 \leq j \leq k$, $\gamma_{j}^{l_{1}}$ is a subpath of $\gamma_{1}^{m_{1}}$, again contrary to $(0)$.

If $l(\gamma)-\left(m_{k} l_{k}+l_{k}^{\prime}\right) \leq i<l(\gamma)-\left(l_{1} l_{k}+l_{k}^{\prime}\right)$, then $\gamma_{1}^{l_{k}}$ is a subpath of $\gamma_{k}^{m_{k}}$, again contrary to $(0)$.

If $i \geq l(\gamma)-\left(l_{1} l_{k}+l_{k}^{\prime}\right)$, then, by (3) above,

contrary to (4) above.

$$
i>l(\gamma)-\frac{m_{1} l_{1}}{2}>l(\gamma)-\frac{l(\gamma)}{2}=\frac{l(\gamma)}{2},
$$

Let $S=\left(\gamma_{1}, \ldots, \gamma_{k}\right)$ and $S^{\prime}=\left(\gamma_{1}^{\prime}, \ldots, \gamma_{k^{\prime}}^{\prime}\right)$ be two sequences of cycles. We say that $S, S^{\prime}$ are equivalent if for each edge $e$, the total number of occurrences of $e$ in $\gamma_{1}, \ldots, \gamma_{k}$ coincides with the total number of occurrences of $e$ in $\gamma_{1}^{\prime}, \ldots, \gamma_{k}^{\prime}$. In particular,

$$
\prod_{i=1}^{k} \mathrm{wt}\left(\gamma_{i}\right)=\prod_{i=1}^{k^{\prime}} \mathrm{wt}\left(\gamma_{i}^{\prime}\right), \quad \sum_{i=1}^{k} l\left(\gamma_{i}\right)=\sum_{i=1}^{k^{\prime}} l\left(\gamma_{i}^{\prime}\right) .
$$

It is easy to see that every cycle $\gamma$ is equivalent to a sequence $S=\left(\gamma_{1}, \ldots, \gamma_{k}\right)$ of simple cycles. In particular, $\operatorname{wps}(\gamma)=\sum_{i=1}^{k}\left(l\left(\gamma_{i}\right) / l(\gamma)\right) \operatorname{wps}\left(\gamma_{i}\right)$.

(1.4) Lemma. Let $A$ be a $\Delta$-form. Let $\gamma_{1}, \ldots, \gamma_{k}$ be a set of precisely one representative from the orbit of each simple cycle. Let $\delta \in \log \Delta_{A}$ and $l \in d_{A} \mathbf{Z}$. Then there exist $u_{i} \in \mathbf{Z}, i=1, \ldots, k$ such that

$$
\delta=\sum_{i=1}^{k} u_{i} \log \mathrm{wt}\left(\gamma_{i}\right)
$$


and

$$
l=\sum_{i=1}^{k} u_{i} l\left(\gamma_{i}\right)
$$

Proof. We may assume $l \geq 0$ (the argument for $l<0$ is similar). First, there exist cycles $\eta, \eta^{\prime}$ such that

$$
d_{A}=l(\eta)-l\left(\eta^{\prime}\right)
$$

Then,

$$
l=l\left(\eta^{l / d_{A}}\right)-l\left(\left(\eta^{\prime}\right)^{l / d_{A}}\right) .
$$

By definition of $\Delta_{A}$ and the fact that $A$ is a $\Delta$-form, there are cycles $\alpha, \alpha^{\prime}$ such that

$$
\left.\delta+\log w \mathrm{t}\left(\left(\eta^{\prime}\right)^{l / d_{A}}\right)\right)-\log \mathrm{wt}\left(\eta^{l / d_{A}}\right)=\log \mathrm{wt}(\alpha)-\log \mathrm{wt}\left(\alpha^{\prime}\right)
$$

and $l(\alpha)=l\left(\alpha^{\prime}\right)$. Thus,

$$
\delta=\log \mathrm{wt}(\alpha)+\log \mathrm{wt}\left(\eta^{l / d_{A}}\right)-\log \mathrm{wt}\left(\alpha^{\prime}\right)-\log \mathrm{wt}\left(\left(\eta^{\prime}\right)^{l / d_{A}}\right)
$$

and

$$
l=l(\alpha)+l\left(\eta^{l / d_{A}}\right)-l\left(\alpha^{\prime}\right)-l\left(\left(\eta^{\prime}\right)^{l / d_{A}}\right) .
$$

Now replace each of $\alpha, \eta^{l / d_{A}}, \alpha^{\prime},\left(\eta^{\prime}\right)^{l / d_{A}}$ by an equivalent sequence of simple cycles.

(1.5) Lemma. Let $P \subset \mathbf{R}^{d}$ be a real polytope of dimension $d$. Let $\left\{w_{1}, \ldots\right.$, $w_{k}$ \} be a finite subset of $P$ which contains $\operatorname{EXT}(P)$. Let $W$ be a set whose closure is contained in int $P$ (here interior means interior in $\mathbf{R}^{d}$ ). Then, $\exists \varepsilon>0$ $\forall w \in W$ we may express $w=\sum_{i=1}^{k} \pi_{i} w_{i}$ where $\sum_{i=1}^{k} \pi_{i}=1$ and each $\pi_{i}>\varepsilon$. Proof. Let $D=\left\{\left(\pi_{1}, \ldots, \pi_{k}\right) \in \mathbf{R}^{k}: \sum_{i=1}^{k} \pi_{i}=1\right.$ and each $\left.\pi_{i} \geq 0\right\}$, the standard simplex.

For $\varepsilon>0$, let

$$
D_{\varepsilon}=\left\{\left(\pi_{1}, \ldots, \pi_{k}\right) \in \mathbf{R}^{k}: \sum_{i=1}^{k} \pi_{i}=1 \text { and each } \pi_{i}>\varepsilon\right\} .
$$

Now, define

$$
\Phi: \mathbf{R}^{k} \rightarrow \mathbf{R}^{d}, \quad\left(\pi_{1}, \ldots, \pi_{k}\right) \mapsto \sum_{i=1}^{k} \pi_{i} w_{i}
$$

Since $\left\{w_{1}, \ldots, w_{k}\right\}$ contains the extreme points of $P, \Phi(D)=P$. Since dimension $(P)=d, \Phi$ is onto $R^{d}$. Since $\Phi$ is linear, $\Phi$ is open. Thus, $\Phi($ int $D)$ is open in $\mathbf{R}^{d}$. Now, since $\Phi$ is continuous, $\Phi($ int $D)$ is dense in $\Phi(D)=P$. So, $\Phi($ int $D)$ is an open, dense, convex subset of $P$. Thus, $\Phi($ int $D)=\operatorname{int} P$.

Now, int $D=\bigcup_{\varepsilon>0} D_{\varepsilon}$. Thus, $\left\{\Phi\left(D_{\varepsilon}\right): \varepsilon>0\right\}$ is an open cover of int $P$. Since $W$ is compact, we get $W \subset \Phi\left(D_{\varepsilon}\right)$ for some $\varepsilon>0$. But this is what we wanted to prove.

Proof of Theorem (1.1). We may assume that $A$ is a $\Delta$-form.

(1) If $\gamma \in \mathscr{C}_{w}$, then $d_{A} / l(\gamma)$ and $l(\gamma) \operatorname{wps}(\gamma)=\log \operatorname{wt}(\gamma) \in \log \Delta_{A}$. Thus, $d(w) / l(\gamma)$. 
(2) We may assume, by attaching a prefix to $\alpha$, that $\alpha$ is a cycle. From (1), it suffices to show that there exists $N$ such that for each $w \in W$, and each $n \in(d(w) Z) \cap[N, \infty)$ there are at least $M$ cycles in $\mathscr{C}_{w, \alpha}$ with length $=n$ and pairwise distinct orbits. Let $\eta$ be a cycle which meets every state of $G(A)$. We may assume that $\underline{i}(\eta)=\underline{i}(\alpha)$. Let $\gamma_{1}, \ldots, \gamma_{k}$ be distinct representatives from the orbits of each simple cycle. We may write $\eta=\eta_{1} \cdots \eta_{k}$ such that $\gamma_{1} \eta_{1} \cdots \gamma_{k} \eta_{k}$ is an allowed cycle. Since $G(A)$ does not consist entirely of a single cycle, $k \geq 2$.

Now, the cycle $\eta \alpha$ is equivalent to a sequence of simple cycles. So,

$$
\mathrm{wt}(\eta \alpha)=\prod_{i=1}^{k} \mathrm{wt}\left(\gamma_{i}\right)^{v_{i}}, \quad l(\eta \alpha)=\sum_{i=1}^{k} v_{i} l\left(\gamma_{i}\right)
$$

for some nonnegative integers $v_{i}$.

Let $W$ be the given closed subset of int $\overline{\mathrm{WPS}}_{A}$. Applying (1.5) to $P \equiv$ closure of $\overline{\mathrm{WPS}}_{A}$ in $R^{d}$, and to the closure of $W$ in $\mathbf{R}^{d}$, there exists $\varepsilon>0$ such that for each $w \in W$, we may express

$$
w=\sum_{i=1}^{k} \pi_{i} \operatorname{wps}\left(\gamma_{i}\right), \quad \sum_{i=1}^{k} \pi_{i}=1 \text { and each } \pi_{i}>\varepsilon .
$$

Let $n$ be large (how large is indicated below and is independent of $w \in W$ ) with $d(w) / n$ i.e., $n w \in \log \Delta_{A}$ and $d_{A} / n$. We will show that $n \in \mathscr{L}\left(\mathscr{C}_{w, \alpha}\right)$.

Let $p_{i}=\left\lfloor\pi_{i} n / l\left(\gamma_{i}\right)\right\rfloor-v_{i}$. (Among other things, $n$ is required to be large enough that each $p_{i}>0$.)

Let $\Theta=\gamma_{1}^{p_{1}} \eta_{1} \gamma_{2}^{p_{2}} \eta_{2} \cdots \gamma_{k}^{p_{k}} \eta_{k} \alpha$.

Let $\delta=n w-\log \mathrm{wt}(\boldsymbol{\Theta}) \in \log \Delta_{A}, l=n-l(\Theta)$. Then, by (5)

$$
\|\delta\|=\left\|\sum_{i=1}^{k}\left(\frac{\pi_{i} n}{l\left(\gamma_{i}\right)}-\left\lfloor\frac{\pi_{i} n}{l\left(\gamma_{i}\right)}\right\rfloor\right) \log \mathrm{wt}\left(\gamma_{i}\right)\right\| \leq \sum_{i=1}^{k}\left\|\log \mathrm{wt}\left(\gamma_{i}\right)\right\| .
$$

So, $\delta$ and $l$ can take on only finitely many values, independent of $n$ and $w$. Thus by (1.4), there exist integers $u_{i}$ such that

$$
\delta=\sum_{i=1}^{k} u_{i} \log \mathrm{wt}\left(\gamma_{i}\right) \quad \text { and } \quad l=\sum_{i=1}^{k} u_{i} l\left(\gamma_{i}\right)
$$

and the $u_{i}$ are bounded above and below independent of $n$ and $w$. Let $\overline{\boldsymbol{\Theta}}=\gamma_{1}^{p_{1}+u_{1}} \eta_{1} \gamma_{2}^{p_{2}+u_{2}} \eta_{2} \cdots \gamma_{k}^{p_{k}+u_{k}} \eta_{k} \alpha$. Then $\log \mathrm{wt}(\overline{\boldsymbol{\Theta}})=n w, l(\overline{\boldsymbol{\Theta}})=n$, and $\alpha$ is a suffix of $\overline{\boldsymbol{\Theta}}$. This shows that $n \in \mathscr{L}\left(\mathscr{C}_{w, \alpha}\right)$, provided that $\overline{\boldsymbol{\Theta}} \in \mathscr{C}$ (i.e., the least period of $\bar{\Theta}$ is $l(\bar{\Theta}))$. But, by (1.3), this is true if the $p_{i}+u_{i}$ are sufficiently large. Since the $u_{i}$ are bounded above and below, and since $\pi_{1}, \ldots, \pi_{k}$ are bounded below by $\varepsilon$, which is independent of $w \in W$, the $p_{i}+u_{i}$ will be large provided only that $n$ is sufficiently large.

So, this produces one cycle, $\bar{\Theta}$, in $\mathscr{C}_{w, \alpha}$ with length $=n$. To produce another such cycle, in a distinct orbit, proceed as follows. Pick a cycle $\alpha^{\prime}$ which has $\alpha$ as a suffix and is not a subpath of

$$
\cdots \gamma_{i} \gamma_{i} \gamma_{i} \eta_{i} \gamma_{i+1} \gamma_{i+1} \gamma_{i+1} \cdots, \quad i=1, \ldots, k-1,
$$

and not a subpath of 
Such a cycle $\alpha^{\prime}$ exists since $G(A)$ does not consist entirely of a single cycle. Now, for $n$ sufficiently large, if $\gamma \in \mathscr{C}_{w, \alpha^{\prime}}, l(\gamma)=n$, then $\gamma$ is not in the orbit of $\overline{\boldsymbol{\theta}}$ (and also $\gamma \in \mathscr{C}_{w, \alpha}$ ). This gives two of the desired cycles. Continue in an analogous way to get the others.

\section{BOUNDARY COMPONENTS}

For a face $F$ of $\overline{\mathrm{WPS}}_{A}, G_{F}=G_{F}(A)$ is the subgraph consisting of all edges that belong to some cycle $\gamma$ with $\operatorname{wps}(\gamma) \in F$. Clearly $G_{F}$ is nonwandering; in [MT1, 3.9], it is shown that a cycle $\gamma$ in $G(A)$ lies in $G_{F}$ if and only if $\operatorname{wps}(\gamma) \in F$. Let $G_{F, i}$ denote the irreducible components of $G_{F}$.

For a subgraph $H$ of $G(A), A_{H}$ denotes the restriction of $A$ to $H$ i.e.,

$$
\left(A_{H}\right)_{I J}=\sum_{\left\{e \in E_{I J}, e \text { in } H\right\}}\left(\mathrm{wt}_{A}(e)\right)^{t}
$$

So, we get the matrix $A_{G_{F}}$ which we abbreviate $A_{F}$. Note that $A_{F}$ defines a nonwandering Markov chain with irreducible components $A_{G_{F, i}}$, which we abbreviate $A_{F, i}$. The correspondence $F \rightarrow\left\{A_{F, i}\right\}$ is called the scaffold of Markov chains associated to $A$.

For two matrices that define the same Markov chain, the affine isomorphism between their polytopes sets up a correspondence between their scaffolds: corresponding matrices $A_{F, i}$ define the same Markov chain. (See [MT1, 3.3(1)].)

The scaffold of Markov chains is a useful invariant of many coding notions (See [MT1, §§4, 5].) Here we refine the scaffold as follows to provide even more information.

Let $\mathscr{B}=\mathscr{B}(A)$ denote the set of Markov chains obtained by starting with $A$ and iterating the scaffold construction i.e., $\mathscr{B}$ is the minimal collection of Markov chains that contains $A$ and contains each $B_{F, i}$ for each $B \in \mathscr{B}$ and each face $F$ of $\overline{\text { WPS }}_{B}$. We call the elements of $\mathscr{B}$ boundary components. Often we will abuse notation by referring to the defining subgraphs (of the Markov chains in $\mathscr{B}$ ) as the elements of $\mathscr{B}$ (i.e., $K$ instead of $A_{K}$ ).

By a chain of boundary components, we mean a sequence of subgraphs

$$
G(A)=G_{0} \supset G_{1} \supset \cdots \supset G_{l}
$$

such that $G_{j+1}=\left(G_{j}\right)_{F_{j+1}, i_{j+1}}$ and $F_{j+1}$ is a face of $\overline{\mathrm{WPS}}_{A_{G_{j}}}$. By definition, every boundary component $K$ is the terminal element of a chain of boundary components (i.e., $K=G_{l}$ as above). Such a chain is called a $K$-chain. A minimal $K$-chain is a chain

$$
G(A)=G_{0} \supset G_{1} \supset \cdots \supset G_{l}=K
$$

such that for every $K$-chain

$$
G(A)=G_{0}^{\prime} \supset G_{1}^{\prime} \supset \cdots \supset G_{l^{\prime}}^{\prime}=K,
$$

$l^{\prime} \geq l$ and $G_{i} \subset G_{i}^{\prime}, i=0, \ldots, l$.

The boundary components have the following properties.

\section{(2.1) Proposition.}

(1) $\mathscr{B}$ is finite.

(2) If, for each face $F, A_{F}$ is irreducible, then $\mathscr{B}=\left\{A_{F}\right\}_{\text {faces } F}$ 
(3) Let $A_{K}, A_{K^{\prime}} \in \mathscr{B}$. If int $\overline{\mathrm{WPS}}_{A_{K}} \cap$ int $\overline{\mathrm{WPS}}_{A_{K^{\prime}}} \neq \varnothing$, and $K$ meets $K^{\prime}$, then $K=K^{\prime}$.

(4) Each boundary component $K$ has a unique minimal $K$-chain.

(5) For each cycle $\gamma$ there is a unique boundary component $K_{\gamma}$ which contains $\gamma$ and satisfies $\operatorname{wps}(\gamma) \in$ int $\overline{\mathrm{WPS}}_{\boldsymbol{A}_{K_{\gamma}}}$.

(6) If $\operatorname{wps}(\gamma)=\operatorname{wps}(\eta)$ and $K_{\gamma}$ meets $K_{\eta}$, then $K_{\gamma}=K_{\eta}$.

(7) Let $A_{K} \in \mathscr{B}$. If $\gamma$ is a cycle that meets $K$ and $\operatorname{wps}(\gamma) \in \overline{\mathrm{WPS}}_{A_{K}}$, then $\gamma$ lies in $K$.

(8) $\mathrm{WPS}_{A}=\bigcup$ int $\overline{\mathrm{WPS}}_{A_{K}}$ over all boundary components $K$.

(9) If either $\operatorname{Rank}\left(\Delta_{A}\right) \leq 1$ or for each $F$ of $\overline{\mathrm{WPS}}_{A} A_{F}$ is irreducible, then $\mathrm{WPS}_{A}=\overline{\mathrm{WPS}}_{A}$.

Proof. (1) Each time that the scaffold construction is iterated and a new boundary component is created, the dimension of the polytope strictly decreases; thus, the iteration can continue only a finite number of times.

(2) Since $\overline{\mathrm{WPS}}_{A_{F}}=F$, each face of $\overline{\mathrm{WPS}}_{A_{F}}$ is a face of $\overline{\mathrm{WPS}}_{A}$. Continue inductively.

(3) It suffices to show $K^{\prime} \subset K$. Let

$$
G(A)=G_{0} \supset G_{1} \supset \cdots \supset G_{l}=K
$$

be a $K$-chain, so, $G_{j+1}=\left(G_{j}\right)_{F_{j+1}, i_{j+1}}$ and $F_{j+1}$ is a face of $\overline{\mathrm{WPS}}_{A_{G_{j}}}$. Fix $w_{0} \in$ int $\overline{\mathrm{WPS}}_{A_{K}} \cap$ int $\overline{\mathrm{WPS}}_{A_{K^{\prime}}}$. We show, inductively, that $K^{\prime} \subset G_{j}$. Clearly $K^{\prime} \subset G_{0}$. So, assume $K^{\prime} \subset G_{j}$ and let $w \in \overline{\mathrm{WPS}}_{A_{K^{\prime}}}$. Since $w_{0} \in$ int $\overline{\mathrm{WPS}}_{A_{K^{\prime}}}$ there is a line segment $L_{w} \subset \overline{\mathrm{WPS}}_{A_{K^{\prime}}}$ such that $w \in L_{w}$ and $w_{0} \in$ int $L_{w}$. Since $w_{0} \in \overline{\mathrm{WPS}}_{A_{K}}, F_{j+1}$ contains $w_{0}$. And since $K^{\prime} \subset G_{j}$, we have $L_{w} \in$ $\overline{\mathrm{WPS}}_{A_{G_{j}}}$. Thus $F_{j+1}$ is a face of the polytope $\overline{\mathrm{WPS}}_{A_{G_{j}}}$, and it contains an interior point of the line segment $L_{w} \in \overline{\mathrm{WPS}}_{A_{G_{j}}}$. Thus $F_{j+1}$ must contain all of $L_{w}$ and hence $w$. Thus, $\overline{\mathrm{WPS}}_{A_{K^{\prime}}} \subset F_{j+1}$. Thus, $K^{\prime}$ is contained in a unique component of $\left(A_{G_{j}}\right)_{F_{j+1}}$. But $G_{j+1}$ is the unique component of $\left(A_{G_{j}}\right)_{F_{j+1}}$ which contains $K$. Since $K$ meets $K^{\prime}$, we get $K^{\prime} \subset G_{j+1}$ as desired.

(4) Inductively assume $K \subset G_{j}$ and define $F_{j+1}$ to be the intersection of all the faces of $\overline{\mathrm{WPS}}_{A_{G_{j}}}$ which contain $\overline{\mathrm{WPS}}_{A_{K}}$. Then $F_{j+1}$ is a face of $\overline{\mathrm{WPS}}_{A_{G_{j}}}$, and there is a unique $i_{j+1}$ such that $\left(G_{j}\right)_{F_{j+1}, i_{j+1}} \supset K$. Let $G_{j+1}=\left(G_{j}\right)_{F_{j+1}, i_{j+1}}$. This defines a sequence of subgraphs which must eventually stabilize i.e., defines a chain:

$$
G(A)=G_{0} \supset G_{1} \supset \cdots \supset G_{l} .
$$

We claim that this is a $K$-chain i.e., $G_{l}=K$. To see this, first note that since the chain stabilized at $G_{l}$, we must have int $\overline{\mathrm{WPS}}_{A_{K}} \cap$ int $\overline{\mathrm{WPS}}_{A_{G_{l}}} \neq \varnothing$. This together with (3) and the fact that $G_{l} \supset K$ yields $G_{l}=K$. To see that this $K$ chain is minimal, inductively apply the fact that if $G^{\prime} \supset G \supset K$ are subgraphs and $F^{\prime}$ is a face of $\overline{\mathrm{WPS}}_{A_{G^{\prime}}}$ such that $F^{\prime} \supset \overline{\mathrm{WPS}}_{A_{K}}$, then there is a face $F$ of $\overline{\mathrm{WPS}}_{A_{G}}$ such that $F^{\prime} \supset F \supset \overline{\mathrm{WPS}}_{A_{K}}$; namely, $F \equiv F^{\prime} \cap \overline{\mathrm{WPS}}_{A_{G}}$.

From the definition it is clear that there can be at most one minimal $K$-chain for each boundary component $K$.

(5) Define $K_{\gamma}$ as follows. Choose any $G_{F, i}$ which contains $\gamma$ and satisfies $\operatorname{wps}(\gamma) \in F$. Now, iterate this procedure starting with $A_{F, i}$ instead of $A$. 
Each time that the procedure is iterated, either $\operatorname{wps}(\gamma) \in \operatorname{int} \overline{\mathrm{WPS}}_{A_{F, i}}$ or the dimension of WPS decreases. So, eventually the former must hold, and this defines $K_{\gamma}$.

For the uniqueness, apply (3).

(6) Apply (3) and (5).

(7) Inductively, one sees that $\gamma$ must lie in each of the subgraphs of any $K$-chain.

(8) From (5), we have wps $(\gamma) \in \operatorname{int} \overline{\mathrm{WPS}}_{A_{K_{\gamma}}}$. This, together with (1.2)(1), yields (8).

(9) If $\operatorname{Rank}\left(\Delta_{A}\right)=1$, then by $[\mathrm{MT} 1,3.4], \operatorname{dim}\left(\overline{\mathrm{WPS}}_{A}\right)=1$. So, by (8),

$$
\mathrm{WPS}_{A}=\operatorname{int} \overline{\mathrm{WPS}}_{A} \cup \partial \overline{\mathrm{WPS}}_{A}=\overline{\mathrm{WPS}}_{A}
$$

If, for each face $F, A_{F}$ is irreducible, then $A_{F}$ is a boundary component and $\overline{\mathrm{WPS}}_{A_{F}}=F$. Thus, by (8)

$$
\mathrm{WPS}_{A}=\bigcup_{\text {faces } \mathrm{F}} \text { int } F=\overline{\mathrm{WPS}}_{A}
$$

(the latter equality comes from the fact that each element of a polytope lies in the interior of some face of the polytope: namely, the intersection of all of the faces that contain the element).

In the remainder of the paper, we will often have occasion to refer to the boundary component $K_{\gamma}$ associated to a cycle $\gamma$ as in (2.1)(5).

(2.2) Example. Let $x, y>0$ s.t. $\log x, \log y$ are independent over $Q$. Define $A$ by the following graph (the labels define $\mathrm{wt}_{A}$ ):

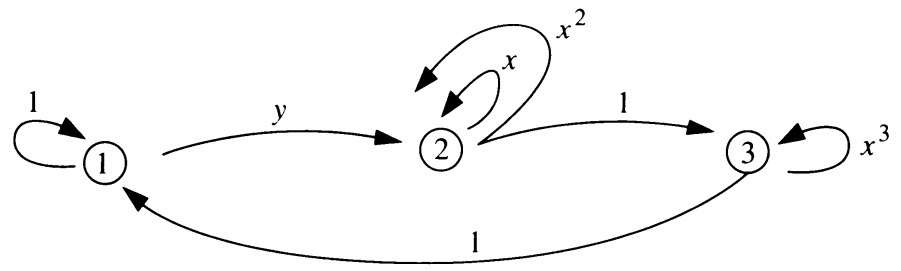

With $\log x, \log y$ as a basis for $\left\langle\mathrm{WPS}_{A}\right\rangle_{Q}$, we identify $\langle\mathrm{WPS}\rangle_{Q} \leftrightarrow Q^{2}$, $\log x \leftrightarrow(1,0)$ and $\log y \leftrightarrow(0,1)$. Then, $\overline{\mathrm{WPS}}_{A}$ is represented as the triangle in $Q^{2}$ with vertices $(0,0),\left(0, \frac{1}{3}\right)$ and $(3,0)$ :

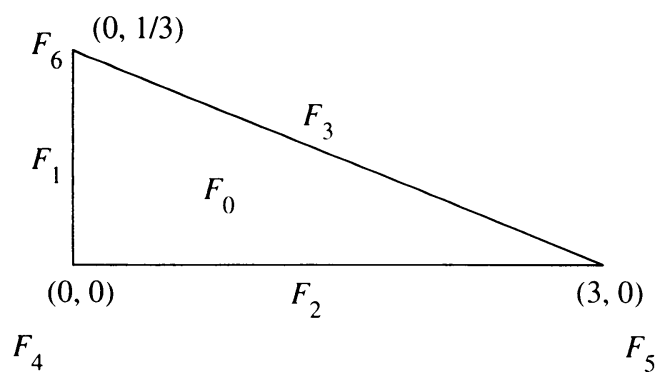


$\overline{\mathrm{WPS}}_{A}=F_{0}$ has three 1 -faces and three 0 -faces. For all these faces $F_{j}$, except $F_{2}, A_{F_{j}}$ is irreducible and does not produce any new boundary components i.e., each boundary component of $A_{F_{j}}$ is some $A_{F_{k}}$. For example, $A_{F_{1}}$ is given by the subgraph consisting of:

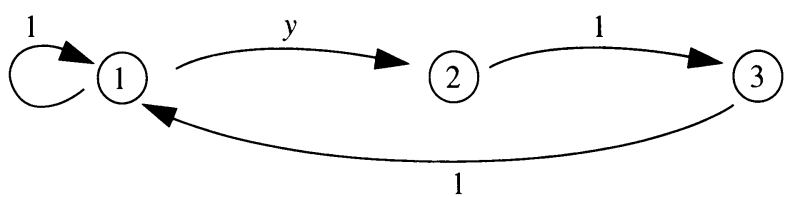

and the boundary components of $A_{F_{1}}$ are $A_{F_{4}}, A_{F_{6}}$. However, $A_{F_{2}}$ has three irreducible components: $A_{F_{4}}, A_{F_{5}}$ and $A_{K}$ where $K$ is the subgraph given by the self-loops with weights $x, x^{2}$, and, $A_{K}$ has two boundary components $A_{K_{1}}, A_{K_{2}}: K_{1}$ is the self-loop with weight $x$, and $K_{2}$ is the self-loop with weight $x^{2}$. Thus, the boundary components are $A_{F_{0}}, A_{F_{1}}, A_{F_{2}}, A_{F_{3}}, A_{F_{4}}$, $A_{F_{5}}, A_{F_{6}}, A_{K}, A_{K_{1}}, A_{K_{2}}$, and all but the last two constitute the scaffold. By (2.1)(8), $\mathrm{WPS}_{A}$ consists of the triangle, $\overline{\mathrm{WPS}}_{A}$, except for the union of two intervals:

$$
\{(t, 0): t \in(0,1) \cup(2,3)\} .
$$

In particular, $\mathrm{WPS}_{A} \neq \overline{\mathrm{WPS}}_{A}$.

For a boundary component $K$, let $\bar{A}_{K}$ be a $\Delta$-form of $A_{K}$. For $w \in$ int $\overline{\mathrm{WPS}}_{A_{K}}$, let $\bar{w}$ denote the image of $w$ via the affine transformation $\overline{\mathrm{WPS}}_{A_{K}}$ $-\overline{\mathrm{WPS}}_{\bar{A}_{K}}$ (as in [MT1, (3.3)(1)]).

For $w \in$ int $\overline{\mathrm{WPS}}_{A_{K}}$, define $d_{A_{K}}(w)=d_{\bar{A}_{K}}(\bar{w})$. For $w \in \mathrm{WPS}_{A}$, let

$$
S(w)=\left\{A_{K} \in \mathscr{B}: w \in \operatorname{int} \overline{\mathrm{WPS}}_{A_{K}}\right\} .
$$

Then, we immediately get the following version of (1.1) for all $w \in \mathrm{WPS}_{A}$.

(2.3) Theorem.

(1) For $w \in \mathrm{WPS}_{A}, \mathscr{L}\left(\mathscr{C}_{w}\right) \subset \bigcup_{K \in S(w)} d_{A_{K}}(w) Z$.

(2) For $w \in \mathrm{WPS}_{A}$, there exists $N_{w} \in \mathbf{N}$ such that

$$
\mathscr{L}\left(\mathscr{C}_{w}\right) \cap\left[N_{w}, \infty\right)=\left(\bigcup_{K \in S(w)} d_{A_{K}}(w) Z\right) \cap\left[N_{w}, \infty\right) .
$$

Proof. Apply (2.1)(5) and (1.1).

Note how (2.3) differs from (1.1) (i.e., how the boundary differs from the interior). For $w \in \mathrm{WPS}_{A}-$ int $\overline{\mathrm{WPS}}_{A}, \mathscr{L}\left(\mathscr{C}_{w}\right)$ is eventually a finite union of periodic sequences (instead of being eventually periodic) and $\mathscr{C}_{w, \alpha}$ may be empty (for instance if $\alpha$ is a cycle and $w$ belongs to some face that does not contain wps $(\alpha))$. In general, there is no uniformity in $N_{w}$ over $\overline{\mathrm{WPS}}_{A}$. Uniformity can fail even for Bernoulli shifts (i.e., Markov chains defined by $1 \times 1$ matrices): 
(2.4) Example. Let $x>0, x \neq 1$. Define $A$ by

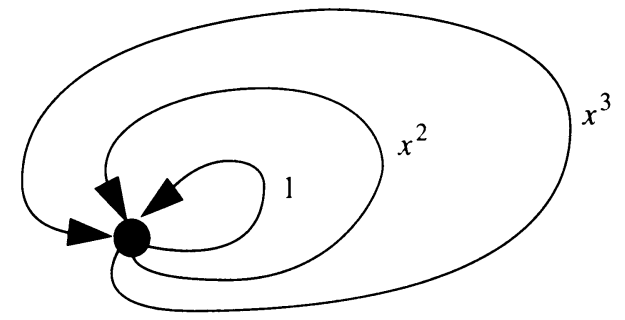

Note that $\Delta_{A}=\left\{x^{n}: n \in Z\right\}$ and $A$ is a $\Delta$-form. Now, with $\{\log x\}$ as basis for $\left\langle\mathrm{WPS}_{A}\right\rangle_{Q} \approx Q$, we can identify

$$
\mathrm{WPS}_{A}=\overline{\mathrm{WPS}}_{A}=[0,3] \cap Q .
$$

Let $N$ be a positive integer and $w=\frac{1}{N}$. So, $w \in \operatorname{int} \overline{\mathrm{WPS}}_{A}$, and so

$$
\bigcup_{K \in S(w)}\left(d_{A_{K}}(w)\right) \mathbf{Z}=d_{A}(w) \mathbf{Z}=N \mathbf{Z}
$$

But since there is no cycle $\gamma$ with $\operatorname{wt}(\gamma)=x, N \notin \mathscr{L}\left(\mathscr{C}_{w}\right)$. So, there is no uniformity in $N_{w}$.

\section{THE INDUCED PERMUTATION OF BOUNDARY COMPONENTS}

Let $\Sigma_{A}$ and $\Sigma_{B}$ be Markov chains. A block isomorphism is a topological conjugacy of the underlying SFT's which is measure-preserving. Given a topological conjugacy, $\varphi$, of the underlying SFT's, for each cycle $\gamma \in \mathscr{C}(A)$, there is a unique cycle, called $\varphi(\gamma) \in \mathscr{C}(B)$ such that $\varphi\left(\gamma^{\infty}\right)=(\varphi(\gamma))^{\infty}$. (Recall that $\mathscr{C}(A), \mathscr{C}(B)$ are the sets of cycles whose least period and length coincide, and $\gamma^{\infty}$ is defined by $\left(\gamma^{\infty}\right)_{i}=e_{i} \bmod n$ where $\gamma=e_{1} \cdots e_{n}$.) Now $\varphi$ is measure-preserving if it is weight-preserving in the sense that for some $a>0, \mathrm{wt}_{A}(\gamma)=a^{l(\gamma)} \mathrm{wt}_{B}(\gamma)$ for each cycle $\gamma \in \mathscr{C}(A)$. (This is well known; see [Ki, JKKMS].) An automorphism of $\Sigma_{A}$ is a self-block isomorphism. An automorphism of the underlying SFT (i.e., a self-topological conjugacy) is measurepreserving if it satisfies the above weight-preserving condition on cycles with $a=1$.

The set of boundary components is ordered by inclusion of the defining graphs.

We first note

(3.1) Proposition. Any automorphism, $\varphi$, of $\Sigma_{A}$ must permute the boundary components in a way that preserves order and minimal $K$-chains.

Proof. Iterate the fact [MT1, 3.13(4)] that $\varphi$ must permute the irreducible components of each $A_{F}$. (This uses the weight-preserving condition above.) Now, apply the definition of boundary component; this gives a permutation of boundary components that clearly preserves the ordering by inclusion. From this, it also follows that it preserves minimal $K$-chains too.

Clearly, if $K, K^{\prime}$ are boundary components, $\varphi$ is an automorphism and $\varphi\left(\Sigma_{A_{K}}\right)=\Sigma_{A_{K^{\prime}}}$, then $\Sigma_{A_{K}}$ and $\Sigma_{A_{K^{\prime}}}$ must be block isomorphic. So, any difference in $\mathrm{WPS}_{A}, \Delta_{A}$ or any other block isomorphism invariant (beta function, 
zeta function, etc.-see [PW, PS]), gives restrictions on the permutations that can be induced by automorphisms. The following example shows how the orderpreserving requirement also gives restrictions.

(3.2) Example. Define $A$ by the following labelled graph where $\log x, \log y$ are independent over $Q$.

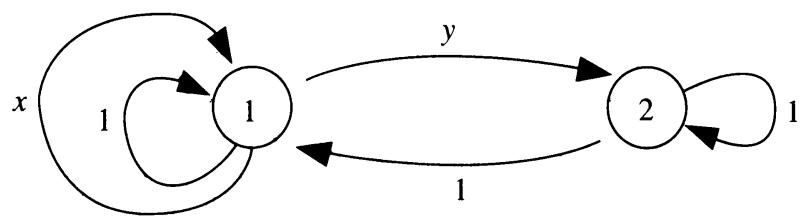

With $\{\log x, \log y\}$ as basis, $\overline{\mathrm{WPS}}_{A}$ is identified as the triangle in $Q^{2}$ with vertices $(0,0),(1,0)$ and $\left(0, \frac{1}{2}\right)$. And $F=\{0\}$ is a face with two block isomorphic components $\Sigma_{A_{K_{1}}}, \Sigma_{A_{K_{2}}}$ :

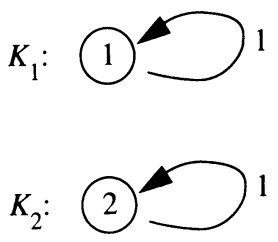

We claim that no automorphism can switch $\Sigma_{A_{K_{1}}}, \Sigma_{A_{K_{2}}}$. Well, for the following boundary component, $\Sigma_{A_{K_{3}}}$,

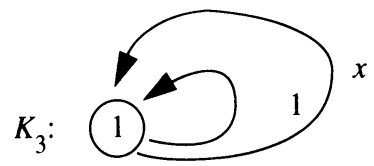

and for any automorphism $\varphi$, we have $\varphi\left(\Sigma_{A_{K_{1}}}\right) \subset \varphi\left(\Sigma_{A_{K_{3}}}\right)$. But since $K_{3}$ is the only boundary component with $\overline{\mathrm{WPS}}=\overline{\mathrm{WPS}}_{A_{K_{3}}}$, we must have $\varphi\left(\Sigma_{A_{K_{3}}}\right)=\Sigma_{A_{K_{3}}}$ and thus $\varphi\left(\Sigma_{A_{K_{1}}}\right) \subset \Sigma_{A_{K_{3}}}$. So, $\varphi\left(\Sigma_{A_{K_{1}}}\right)=\Sigma_{A_{K_{1}}}$.

We do not know a complete and effective set of conditions for a permutation of boundary components to be realized by an automorphism. However, we do give a necessary condition, in terms of shift equivalence, which can actually be checked in some cases. For this, we first change our viewpoint on the defining matrices for Markov chains.

Let $\bar{A}$ be a matrix over $Z^{+}\left[\bar{x}_{1}^{ \pm} \ldots, \bar{x}_{N}^{ \pm}\right]$where $\bar{x}_{1}, \ldots, \bar{x}_{n}$ are commuting indeterminates. Such a matrix $\bar{A}$ together with a set of numbers $x_{1}, \ldots, x_{n} \in$ $\mathbf{R}^{+}$determines a $Z^{+}[\exp ]-$ matrix $A$ : simply replace each monomial $\prod_{i=1}^{n} \bar{x}_{i}^{w_{i}}$, with $\prod_{i=1}^{n}\left(x_{i}^{w_{i}}\right)^{t}$.

Now, conversely any matrix $A$ over $Z^{+}$[exp] determines such an $\bar{A}$ as follows. Choose a basis $\left\{x_{1}, \ldots, x_{n}\right\}$ for the multiplicative group generated by the weights of all the edges. For example, if $A$ were a $\Delta$-form, then $\left\{x_{1}, \ldots, x_{n}\right\}$ 
would be a basis for $\Delta_{A}$. Now, for edge $e$, there is a unique expression

$$
\mathrm{wt}_{A}(e)=\prod_{i=1}^{n} x_{i}^{w_{i}(e)}, \quad w_{i}(e) \in \mathbf{Z} .
$$

Let $\bar{A}$ be the matrix over $Z^{+}\left[\bar{x}_{1}^{ \pm}, \ldots, \bar{x}_{n}^{ \pm}\right]$defined by

$$
\bar{A}_{I J}=\sum_{e \in E_{I J}} \prod_{i=1}^{n} \bar{x}_{i}^{w_{i}(e)} \text {. }
$$

Note that $\bar{A}$ depends on the choice of basis only up to an affine transformation of the exponents in $\mathbf{Z}^{n}$. We call $\bar{A}$ a polynomial representation of $A$.

Of course, $A$ can be recovered from $\bar{A}$ and the numbers $x_{1}, \ldots, x_{n}$.

For $p, q \in Z\left[\bar{x}_{1}^{ \pm}, \ldots, \bar{x}_{n}^{ \pm}\right], p \geq q$ means $p-q \in Z^{+}\left[\bar{x}_{1}^{ \pm}, \ldots, \bar{x}_{n}^{ \pm}\right]$.

For matrices, $\bar{A}, \bar{B}$ over $Z^{+}\left[\bar{x}_{1}^{ \pm}, \ldots, \bar{x}_{n}^{ \pm}\right]$, an elementary strong shift equivalence (elementary SSE) is a pair of matrices $(R, S)$ over $Z^{+}\left[\bar{x}_{1}^{ \pm}, \ldots, \bar{x}_{n}^{ \pm}\right]$ satisfying

$$
\bar{A}=R S, \quad \bar{B}=S R .
$$

As in simpler categories (see [Wi]), one can define a set of block isomorphisms, $c(R, S)$, from $\Sigma_{A}$ to $\Sigma_{B}(\bar{A}, \bar{B}$ are polynomial representations of $A, B)$ that come from the elementary SSE $(R, S)$, as follows. (Sometimes we use $c(R, S)$ to denote this set of automorphisms and at other times use $c(R, S)$ to denote an element of the set.)

For a matrix $R$ over $Z^{+}\left[\bar{x}_{1}^{ \pm}, \ldots, \bar{x}_{n}^{ \pm}\right]$, let $M_{I J}(R)=\left\{(u, i): 1 \leq i \leq c_{u}\right\}$ where

$$
R_{I J}=\sum_{\{\text {monomials } u\}} c_{u} u, \quad c_{u} \in \mathbf{Z}^{+}
$$

So, $M_{I J}(R)$ represents the monomials in $R_{I J}$ with multiplicity. For $(u, i) \in$ $M_{I J}(R)$, write $\underline{i}(u, i)=I, \underline{t}(u, i)=J$ and $\mathrm{wt}_{R}(u, i)=u$. Likewise for $S$. Now if $(R, S)$ is an elementary SSE, then we can identify $E_{I J}(A)$ with $\bigcup_{\bar{I} \in S(B)} M_{I \bar{I}}(R) \times M_{\bar{I} J}(S)$ via a bijection, which we denote

$$
e \leftrightarrow(r(e), s(e))
$$

such that $\mathrm{wt}_{A}(e)=\mathrm{wt}_{R}(r(e)) \mathrm{wt}_{S}(s(e))$. Likewise, $E_{I J}(B)$ can be identified with $\bigcup_{\bar{I} \in S(A)} M_{I \bar{I}}(S) \times M_{\bar{I} J}(R)$ via a bijection which we denote

$$
e \leftrightarrow\left(s^{*}(e), r^{*}(e)\right)
$$

Note that if $\underline{t}(e)=\underline{i}(f)$, then $(s(e), r(f))=\left(s^{*}\left(e^{\prime}\right), r^{*}\left(e^{\prime}\right)\right)$ for a unique $e^{\prime}$. Now, define $c(R, S): \Sigma_{A_{0}} \rightarrow \Sigma_{B_{0}}$ as follows. Let $\ldots e_{-1} e_{0} e_{1} \cdots \in \Sigma_{A_{0}}$. Let $e_{i}^{\prime}$ be defined by $\left(s^{*}\left(e_{i}^{\prime}\right), r^{*}\left(e_{i}^{\prime}\right)\right)=\left(s\left(e_{i}\right), r\left(e_{i+1}\right)\right)$. Then $c(R, S)\left(\cdots e_{-1} e_{0} e_{1} \cdots\right)=$ $\cdots e_{-1}^{\prime} e_{0}^{\prime} e_{1}^{\prime} \cdots$.

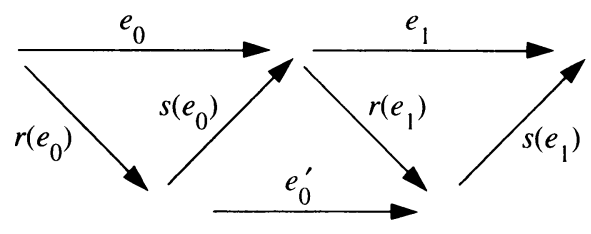


The map $c(R, S)$ depends on the bijections chosen.

Note that the identity belongs to $c(I, \bar{A})$; by analogy with the topological setting, we call the automorphisms that are block isomorphic to the automorphisms in $c(I, \bar{A})$ simple automorphisms (see [N]).

Also, the shift $\sigma_{A_{0}}$ belongs to $c(\bar{A}, I)$. Unfortunately, $\sigma_{A_{0}}^{-1}$ cannot be represented as a $c(R, S)$. However, we have (see Parry-Williams [PW], Parry-Tuncel [PT2], and Wagoner [Wa1, Wa2]):

(3.3) Proposition. Let $\varphi$ be an automorphism of $\Sigma_{A}$. Let $\bar{A}$ be a polynomial representation of $A$. Then, there is a sequence of matrices $R_{i}, S_{i}$ over $Z^{+}\left[\bar{x}_{1}^{ \pm}, \ldots, \bar{x}_{n}^{ \pm}\right]$such that

$$
\begin{aligned}
& \bar{A}=R_{1} S_{1} \\
& S_{1} R_{1}=R_{2} \quad S_{2} \\
& S_{L-1} \quad R_{L-1}=R_{L} \quad S_{L} \\
& S_{L} \quad R_{L}=\bar{A}
\end{aligned}
$$

and $\varphi \circ \sigma_{A_{0}}^{l}=c\left(R_{L}, S_{L}\right) \cdots c\left(R_{1}, S_{1}\right)$ for some $l \geq 0$.

The chain of matrices $\left(R_{i}, S_{i}\right)$ satisfying the equations of (3.3) is called a (self) strong shift equivalence (SSE). A pair $(R, S)$ is called a (self) shift equivalence (SE) (with lag $L$ ) of $A$ if it satisfies:

$$
\bar{A} R=R \bar{A}, \quad S \bar{A}=\bar{A} S, \quad R S=\bar{A}^{L}, \quad S R=\bar{A}^{L} .
$$

Note that any automorphism $\varphi$ of $\Sigma_{A}$ naturally determines an automorphism $\varphi^{(i)}$ of $\Sigma_{A^{i}}$, for each $i$. We say that an SE $(R, S)$ is induced by $\varphi$ if $\varphi^{(L)} \in$ $c(R, S), L=$ lag of $(R, S)$. Note that if $\gamma$ is a path with length equal to the lag $L$ of the SE induced by $\varphi$, then

$$
\mathrm{wt}_{\bar{A}}(\gamma) \leq R_{\underline{\underline{i}}(\gamma), \underline{i}(\varphi(\gamma))} \cdot S_{\underline{i}(\varphi(\gamma)), \underline{t}(\gamma)}
$$

where $\underline{i}(\varphi(\gamma))$ is taken to be the initial state of $(\varphi(z))_{0}, z_{0} \cdots z_{L-1}=\gamma$. This makes sense since the length of $\gamma$ equals the lag of the SE.

It is immediate from the definitions that the SSE, in (3.3), determines an SE $(R, S): R=R_{1} \cdots R_{L}, S=S_{L} \cdots S_{1}$ induced by $\varphi \circ \sigma_{A_{0}}^{l}$.

The induced SE is unique in the following sense: if $(R, S)$ and $\left(R^{\prime}, S^{\prime}\right)$ are SE's induced by the same $\varphi$ with the same lag, then for some monomial $u$

$$
R^{\prime}=u R, \quad S^{\prime}=u^{-1} S .
$$

This follows from Wagoner [Wa2]. For completeness we give a proof in Appendix $A$. Note that since $c(I, \bar{A})=$ identity, if an $\operatorname{SE}(R, S)$ is induced by an automorphism $\varphi$, then there are SE's of all sufficiently large lags induced by $\varphi$; namely, $\left(R, S \bar{A}^{i}\right)$. And if $(R, S)$ and $\left(R^{\prime}, S^{\prime}\right)$ are SE's induced by the same $\varphi$, then (assuming the lag of $\left(R^{\prime}, S^{\prime}\right)$ is at least the lag of $(R, S)$ ), we get for some monomial $u$

$$
R^{\prime}=u R, \quad S^{\prime}=u^{-1} S \bar{A}^{L}
$$

where $L \geq 0$ is the difference in the lags.

For a monomial $u=\prod_{i=1}^{n} \bar{x}_{i}^{w_{i}}$, let $\log (\dot{u})$ denote the vector $\left(w_{1}, \ldots, w_{n}\right)$. Abusing notation, by $\varphi(K)$ we mean the boundary component such that $\varphi\left(\Sigma_{A_{K}}\right)$ $=\Sigma_{A_{\varphi(K)}}$, where $K$ is a boundary component and $\varphi$ an automorphism. 
(3.4) Proposition. Let $\varphi$ be an automorphism and $K, K^{\prime}$ be boundary components. Then, $\varphi(K)=K^{\prime}$ iff $\overline{\mathrm{WPS}}_{A_{K}}=\overline{\mathrm{WPS}}_{A_{K^{\prime}}}$ and there is an $S E(R, S)$ induced by $\varphi \circ \sigma_{A_{0}}^{l}$, some $l$, and states $I \in S\left(A_{K}\right), J \in S\left(A_{K^{\prime}}\right)$ and a monomial $u$ such that

and

$$
u \leq R_{I J} S_{J I}
$$

$$
\frac{\log u}{L} \in \overline{\mathrm{WPS}}_{A_{K}}, \quad L=\text { lag of }(R, S) \text {. }
$$

Moreover, this condition must hold for all $S E$ 's $(R, S)$ whose lag is the length of some cycle in $K$.

Proof. Only if: Clearly $\overline{\mathrm{WPS}}_{A_{K}}=\overline{\mathrm{WPS}}_{A_{K^{\prime}}}$. By (3.3) and the remarks above, for some $l \geq 0, \varphi \circ \sigma_{A_{0}}^{l}$ induces SE's of all sufficiently long lags-in particular, the lag, $L$, can be chosen to equal the length of a cycle $\gamma$ in $K$.

Let $I=\underline{i}(\gamma)$. Since $\left(\varphi \circ \sigma_{A_{0}}^{l}\right)^{(L)} \in c(R, S)$ and $\varphi \circ \sigma_{A_{0}}^{l}(K)=K^{\prime}$,

$$
\mathrm{wt}_{\bar{A}}(\gamma) \leq R_{I J} S_{J I}
$$

for some $J \in S\left(A_{K^{\prime}}\right)$. This establishes the "only if" and the "moreover".

If: Since $u \leq R_{I J} S_{J I}$, there is a cycle $\gamma$ with $\underline{i}(\gamma)=I, \underline{i}(\varphi(\gamma))=J$, $l(\gamma)=L$ and $\mathrm{wt}_{\bar{A}}(\gamma)=u$ (see $(\dagger)$ above). By $(2.1)(7), \gamma$ lies in $K$, and thus $\varphi \circ \sigma_{A_{0}}^{l}(K)$ contains the state $J$. Thus, $\varphi(K)$ meets $K^{\prime}$. This, together with $\overline{\mathrm{WPS}}_{A_{\varphi(K)}}=\overline{\mathrm{WPS}}_{A_{K}}=\overline{\mathrm{WPS}}_{A_{K^{\prime}}}$ and $(2.1)(3)$ gives $\varphi(K)=K^{\prime}$ as desired.

This result gives a way of deciding, for a given automorphism $\varphi$, what the induced permutation of boundary components is. It also gives a necessary condition for the existence of an automorphism that maps one boundary component to another.

(3.5) Example. Let $A$ be defined by the graph

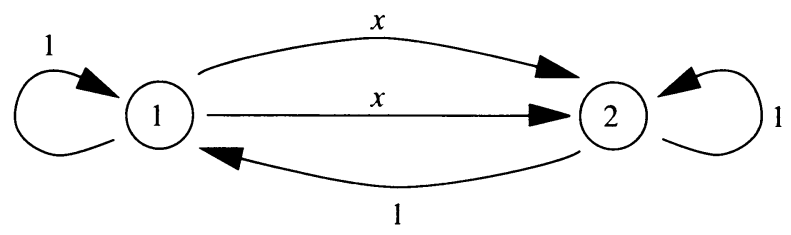

(Here $x>0, x \neq 1$.) Then, $\overline{\mathrm{WPS}}_{A}$ is the convex hull of $\left\{0, \frac{\log x}{2}\right\}$. Let $F$ be the face $\{0\}$. Then $A_{F}$ has two components $A_{F_{1}}, A_{F_{2}}$. While $\Sigma_{A_{F_{1}}}, \Sigma_{A_{F_{2}}}$ are block isomorphic, we claim that there is no automorphism which switches them (and one cannot tell this by simply looking at the boundary components and their ordering). If there were such an automorphism, then by (3.4), there would be an $\mathrm{SE}(R, S)$ of

$$
\bar{A}=\left[\begin{array}{cc}
1 & 2 \bar{x} \\
1 & 1
\end{array}\right]
$$

such that $1 \leq R_{12} S_{21}$ and thus there would be monomials $u \leq R_{12}, u^{\prime} \leq S_{21}$, such that $1=u \cdot u^{\prime}$. Now, a straightforward computation shows that any $R$ which satisfies $\bar{A} R=R \bar{A}$ must be of the form

$$
R=\left[\begin{array}{cc}
a & 2 b \bar{x} \\
b & a
\end{array}\right], \quad a, b \in Z^{+}\left[\bar{x}^{ \pm}\right]
$$


So, $u \leq R_{12}=2 b \bar{x}$, and thus $2 u \leq R_{12}$, and thus

$$
2=2 u \cdot u^{\prime} \leq R_{12} S_{21} \leq\left(\bar{A}^{L}\right)_{11} .
$$

But the constant term of $\left(\bar{A}^{L}\right)_{11}$ is 1 .

As usual, we say that $A$ is mixing if $A_{0}$ is mixing (i.e., if $A_{0}^{n}>0$ for some $n$ ). If $A$ is not mixing, then $S(A)$ breaks up into "cyclically moving subsets" $C_{0}, \ldots, C_{d_{A}-1}$; in particular, letting $\Sigma_{i}=\left\{x \in \Sigma_{A_{0}}: \underline{i}\left(x_{0}\right) \in C_{i}\right\}$, $\sigma_{A_{0}}\left(\Sigma_{i}\right)=\Sigma_{i+1}$. (See [AM, p. 16].)

The boundary components of $A^{m}$ may be slightly different from the boundary components of $A$. For example, if $d_{A_{K}} \mid m$, each boundary component $K$ gives rise to $d_{A_{K}}$ boundary components of $A^{m}$ of period 1 . (These come from the cyclically moving subsets of $K$.) Let $\pi$ be a permutation of $\mathscr{B}(A)$. A permutation $\pi^{(m)}$ of $\mathscr{B}\left(A^{m}\right)$ is consistent with $\pi$ if whenever $K \in \mathscr{B}(A)$ gives rise to $K_{0}, \ldots, K_{d-1} \in \mathscr{B}\left(A^{m}\right)$, then $\pi^{(m)}\left(K_{i}\right)=K_{i_{0}+i}^{\prime}, i=0, \ldots, d-1$, where $K_{0}^{\prime}, \ldots, K_{d-1}^{\prime} \in \mathscr{B}\left(A^{m}\right)$ are the boundary components arising from $\pi(K)$ and $i_{0}$ is some element of $\{0, \ldots, d-1\}$. The proof of $(3.4)$ and arguments in [BMT, 3.2 and MT1, 4.8] yield the following (whose proof we leave to the reader):

(3.6) Proposition. Let $\pi$ be a permutation of the boundary components of $A$. For sufficiently large $m$, there is an automorphism of $\Sigma_{A^{m}}$ which realizes a permutation consistent with $\pi$ iff $\overline{\mathrm{WPS}}_{A_{K}}=\overline{\mathrm{WPS}}_{\boldsymbol{A}_{\boldsymbol{\pi}(K)}}$ for each boundary component $K$ and there is an $S E(R, S)$ and states $I_{K} \in S\left(A_{K}\right), J_{K} \in S\left(A_{\pi(K)}\right)$ and a monomial $u_{K}$ such that

$$
u_{K} \leq R_{I_{K} J_{K}} S_{J_{K} I_{K}}
$$

and

$$
\frac{\log u_{K}}{L} \in \overline{\mathrm{WPS}}_{A_{K}}, \quad L=\text { lag of }(R, S) .
$$

(For the proof of this, it helps to use the fact that for a cycle $\gamma$ and a positive integer $L, \operatorname{wps}_{A^{L}}(\gamma)=L \operatorname{wps}_{A}(\gamma)$.)

For a matrix $\bar{A}$ over $Z^{+}\left[\bar{x}_{1}^{ \pm}, \ldots, \bar{x}_{n}^{ \pm}\right]$, the dimension module $M_{\bar{A}}$ is the $Z\left[\bar{x}_{1}^{ \pm}, \ldots, \bar{x}_{n}^{ \pm}\right]$-module defined by the direct limit:

$$
M_{\bar{A}}=\underset{\overrightarrow{\bar{A}}}{\lim }\left(Z\left[\bar{x}_{1}^{ \pm}, \ldots, \bar{x}_{n}^{ \pm}\right]\right)^{\alpha}
$$

where $\bar{A}$ is $\alpha \times \alpha$. See Tuncel [T2] for an interpretation of $M_{A}$ in terms of a natural equivalence relation on open-closed sets in unstable manifolds. The shift map is reflected by the module automorphism:

$$
\widehat{A}: M_{\bar{A}} \rightarrow M_{\bar{A}}, \quad \widehat{A}([\bar{v}, i])=[\bar{v} \bar{A}, i]
$$

(where $[\bar{v}, i]$ denotes the equivalence class of the " $i$ th copy" of $\bar{v}$ in $\left.\left(Z\left[\bar{x}_{1}^{ \pm}, \ldots, \bar{x}_{n}^{ \pm}\right]\right)^{\alpha}\right)$. Let $\varphi$ be an automorphism of $\Sigma_{A}$ and $\bar{A}$ a polynomial representation of $A$. Then $\varphi \circ \sigma_{A_{0}}^{l}$ induces an SE $(R, S)$ of $\bar{A}$ for some $l \geq 0$. Define the module automorphism $\Pi(\varphi): M_{\bar{A}} \rightarrow M_{\bar{A}}$ by

$$
\Pi(\varphi)([\bar{v}, i])=\widehat{A}^{-l}([\bar{v} R, i])
$$


The automorphism $\Pi(\varphi)$ is well-defined (i.e., independent of $l$ and the particular induced SE), up to composition with a scalar automorphism i.e., an automorphism of the form

$$
[\bar{v}, i] \mapsto[u \bar{v}, i]
$$

for some monomial $u$. This follows from the sense in which the induced SE is unique; see Appendix A.

The map $\Pi$ from automorphisms of $\Sigma_{A}$ to automorphisms of $M_{A}$ is called the dimension representation. The dimension representation is a group homomorphism (the group operation here is composition). As in [Wa2], we say that $\varphi$ is inert if $\Pi(\varphi)$ is a scalar automorphism. By replacing $(R, S)$ by $\left(u R, u^{-1} S\right)$, for appropriate $u$, we may assume that $\Pi(\varphi)=$ identity whenever $\varphi$ is inert. We remark that the inert property does not depend on the particular matrix $A$, used to define the Markov chain, nor on the particular polynomial representation. Also, compositions of inert automorphisms are inert. And inertness is a block isomorphism invariant (i.e., if $\psi=\pi \circ \varphi \circ(\pi)^{-1}$, for some block isomorphism $\pi$, and $\varphi$ is inert, then so is $\psi)$. So the automorphisms $c(I, \bar{A})$, and therefore the simple automorphisms, are inert. These facts are contained in [Wa2].

Inert automorphisms have played a significant role in the theory of automorphisms of SFT's, and they can be expected to play a similar role for automorphisms of Markov chains.

The following is a generalization of the corresponding result for automorphisms of SFT's. It is a straightforward consequence of the definitions.

(3.7) Lemma. An automorphism $\varphi$ is inert iff for some $l \geq 0$, there is an $S E$ $(R, S)$ induced by $\varphi \circ \sigma_{A_{0}}^{l}$ such that $R \bar{A}^{m}=\bar{A}^{m+l}$, for some (and hence all sufficiently large) $m$.

(3.8) Proposition. If $\varphi$ is an inert automorphism of $\Sigma_{A}$, then $\varphi$ must fix each $\Sigma_{i}$ (where the $\Sigma_{i}$ are as defined above from the cyclically moving subsets $C_{i}$.)

Proof. There is an SE $(R, S)$ induced by $\varphi \circ \sigma_{A_{0}}^{l}$, some $l \geq 0$, such that $R \bar{A}^{m}=\bar{A}^{m+l}$, for large $m$. Then $\varphi \circ \sigma_{A_{0}}^{l}\left(\Sigma_{i}\right)=\Sigma_{j}$, some $j$. Let $I \in C_{i}$. Then by definition of induced SE, $R_{I J} \neq 0$ for some state $J \in C_{j}$ (see (†)). Choose $m$ such that $\bar{A}_{J J}^{m} \neq 0$. Of course, $m$ is a multiple of $d_{A}$. Since $R_{I J}\left(\bar{A}^{m}\right)_{J J} \leq\left(\bar{A}^{m+l}\right)_{I J}$ there is a path from $I$ to $J$ of length $m+l$. Thus, $j=i+l\left(\bmod d_{A}\right)$. So, $\varphi \circ \sigma_{A_{0}}^{l}\left(\Sigma_{i}\right)=\Sigma_{i+l}$. So, $\varphi\left(\Sigma_{i}\right)=\Sigma_{i}$ as desired.

It will be convenient to consider polynomial representations $\bar{A}$ that are adapted to a given face as follows: For any proper face $F$, we may assume, by making an affine transformation of $\left\langle\mathrm{WPS}_{A}\right\rangle_{Q} \approx Q^{n}$, that

$$
\overline{\mathrm{WPS}}_{A} \subset\left\{q \in Q^{n}: q_{n} \geq 0\right\}
$$

and

$$
F=\overline{\mathrm{WPS}}_{A} \cap\left\{q \in Q^{n}: q_{n}=0\right\} .
$$

Thus, each cycle $\gamma$ in $G(A)$ satisfies

$$
\mathrm{wt}_{\bar{A}}(\gamma)=\prod_{i=1}^{n} \bar{x}_{i}^{w_{i}}, \quad w_{n} \geq 0,
$$

and $\gamma$ lies in $G_{F}$ iff $w_{n}=0$. 
By conjugating $\bar{A}$ by some diagonal matrix with single monomial entries, we may assume that $\bar{A}$ is over $Z^{+}\left[\bar{x}_{i}^{ \pm}, \ldots, \bar{x}_{n-1}^{ \pm}, \bar{x}_{n}\right]$ and thus $\bar{A}_{F}$ is over $Z^{+}\left[\bar{x}_{i}^{ \pm}, \ldots, \bar{x}_{n-1}^{ \pm}\right]$: Namely, let $D_{I I}=\bar{x}_{n}^{a_{I}}$ where, fixing some arbitrary state $I_{0}$,

$$
a_{I}=-\min \left(\left(\log \mathrm{wt}_{\bar{A}}(\gamma)\right)_{n}\right)
$$

the minimum being taken over all paths $\gamma$ from $I_{0}$ to $I$. The reader may check that $D^{-1} \bar{A} D$ indeed satisfies the above.

When $\bar{A}$ is in this form we say that it is $F$-adapted.

(3.9) Theorem. Let $\varphi$ be an inert automorphism. Then, for each boundary component $K, \varphi(K)=K$ and $\left.\varphi\right|_{\Sigma_{A_{K}}}$ is inert.

Proof. It suffices to prove this for components of each $G_{F}$. We may assume that $\bar{A}$ is $F$-adapted. Let $\mathscr{M}$ be the set of monomials in the variables $\bar{x}_{1}, \ldots, \bar{x}_{n-1}$. Now, there is an $\operatorname{SE}(R, S)$ of $\bar{A}$ induced by $\varphi \circ \sigma_{A_{0}}^{l}$, some $l$, such that

$$
R \bar{A}^{m}=\bar{A}^{m+l}
$$

for large $m$. Fix such an $m$.

We

Claim. $R_{I J}, S_{I J}$ are over $Z^{+}\left[\bar{x}_{1}^{ \pm}, \ldots, \bar{x}_{n-1}^{ \pm}, \bar{x}_{n}\right]$ if either $I$ or $J$ belongs to $S\left(A_{F}\right)$.

To see this, first suppose $J \in S\left(A_{F}\right)$. Let $\gamma$ be a path in $G_{F}$ such that $\underline{i}(\gamma)=J$ and $l(\gamma)=m$. Then

$$
R_{I J} \mathrm{wt}_{\bar{A}}(\gamma) \leq\left(\bar{A}^{m+l}\right)_{I, \underline{t}(\gamma)}
$$

But since $\bar{A}$ is $F$-adapted, $R_{I J}$ is over $Z^{+}\left[\bar{x}_{1}^{ \pm}, \ldots, \bar{x}_{n-1}^{ \pm}, \bar{x}_{n}\right]$. So, we get the claim for $R_{I J}$. Using the SE equations, together with (6), we get

$$
\bar{A}^{m} R=\bar{A}^{m+l}, \quad \bar{A}^{m+l} S=S \bar{A}^{m+l}=\bar{A}^{m+L}
$$

(where $L$ is the lag of the $\mathrm{SE}$ ), and so the same argument gives the entire claim.

Let $K$ be a component of $G_{F}$. We will show that there is a cycle $\eta$ which lies in $G_{F}$ and meets $K, \varphi(K), \varphi^{2}(K), \ldots$ and therefore $K$ is $\varphi$-invariant. Let $\gamma$ be a path in $K$ such that $L(\gamma)=L$. Then, by definition of induced SE,

$$
\mathrm{wt}_{\bar{A}}(\gamma) \leq R_{\underline{\underline{i}}(\gamma), \underline{i}(\varphi(\gamma))} S_{\underline{i}(\varphi(\gamma)), \underline{t}(\gamma)} .
$$

But since $\bar{A}$ is $F$-adapted, wt $\overline{-}_{\bar{A}}(\gamma) \in \mathscr{M}$. And since $R_{\underline{i}(\gamma), \underline{i}(\varphi(\gamma))}, S_{\underline{i}(\varphi(\gamma)), \underline{t}(\gamma)}$ both belong to $Z^{+}\left[\bar{x}_{1}^{ \pm}, \ldots, \bar{x}_{n-1}^{ \pm}, \bar{x}_{n}\right]$ (by the claim above), $\mathrm{wt}_{\bar{A}}(\gamma)$ may be written as the product of two monomials in $\mathscr{M}$, one of which, called $u$, satisfies

$$
u \leq R_{\underline{i}(\gamma), \underline{i}(\varphi(\gamma))} \text {. }
$$

Now, let $\bar{\gamma}$ be any path of length $m$ in $\varphi(K)$ with $\underline{i}(\bar{\gamma})=\underline{i}(\varphi(\gamma))$. Then,

$$
\mathrm{wt}_{\bar{A}}(\bar{\gamma}) \leq\left(\bar{A}^{m}\right)_{\underline{i}(\varphi(\gamma)), \underline{t}(\bar{\gamma})} .
$$

Thus,

$$
\begin{aligned}
u \cdot \mathrm{wt}_{\bar{A}}(\bar{\gamma}) \leq R_{\underline{\underline{i}}(\gamma), \underline{\underline{i}}(\varphi(\gamma))}\left(\bar{A}^{m}\right)_{\underline{\underline{i}}(\varphi(\gamma)), \underline{t}(\bar{\gamma})} \\
\leq\left(R \bar{A}^{m}\right)_{\underline{\underline{\gamma}}(\gamma), \underline{\underline{t}}(\bar{\gamma})}=\left(\bar{A}^{m+l}\right)_{\underline{\underline{i}}(\gamma), \underline{t}(\bar{\gamma})} .
\end{aligned}
$$


So, there is a path from $K$ to $\varphi(K)$ with weight in $\mathscr{M}$. Applying this same argument to $\varphi^{j}(K)$ instead of $K$, we get a path from $\varphi^{j}(K)$ to $\varphi^{j+1}(K)$ with weight in $\mathscr{M}$. Thus, there is a cycle, with weight in $\mathscr{M}$, which meets each $\varphi^{j}(K)$, as desired.

Finally, we need to show that $\left.\varphi\right|_{\Sigma_{A_{K}}}$ is inert. We construct an SE $\left(R_{K}, S_{K}\right)$ induced by $\varphi \circ \sigma_{A_{0}}^{l} \mid \Sigma_{A_{K}}$ and show $R_{K} \bar{A}_{K}^{m}=\bar{A}_{K}^{m+l}$, for some polynomial representation $\bar{A}_{K}$ of $A_{K}$. (Here $m$ and $l$ are the same as in (6).) We let $\bar{A}_{K}$, $R_{K}, S_{K}$ (resp.) be the matrices, indexed by the states of $K$, with entries equal to the parts of $\bar{A}_{I J}, R_{I J}, S_{I J}$ (resp.) that involve monomials in $\mathscr{M}$, e.g., if we write $S_{I J}=\sum_{\{\text {monomials } u\}} a_{u} u, a_{u} \in \mathbf{N}$, then $\left(S_{K}\right)_{I J}=\sum_{\{\text {monomials } u \in \mathscr{M}\}} a_{u} u$.

Note that $\bar{A}_{K}$ is indeed a polynomial representation of $A_{K}$. We first show

$$
R_{K} S_{K}=\bar{A}_{K}^{L}
$$

( $L$ is the lag of the SE $(R, S))$. Well, clearly,

$$
R_{K} S_{K} \leq \bar{A}_{K}^{L}
$$

Now, let $u \leq\left(\bar{A}_{K}\right)_{I J}^{L}$ (of course, $I, J \in S\left(A_{K}\right)$ ). Then since $\bar{A}_{K}^{L} \leq \bar{A}^{L}=R S$, there is a state $\bar{I} \in S(A)$ and monomials $u_{1}, u_{2}$ such that

$$
u=u_{1} u_{2}, \quad u_{1} \leq R_{\overline{I I}}, \quad u_{2} \leq S_{\bar{I} J} .
$$

To show that $\bar{A}_{K}^{L} \leq R_{K} S_{K}$, it suffices to show that

$$
\bar{I} \in S\left(A_{K}\right)
$$

and

$$
u_{1}, u_{2} \in \mathscr{M}
$$

For $(*)$, note that by definition of induced SE, there is a path $\gamma$ in $K$ from $I$ to $J$ such that $\bar{I}=\underline{i}\left(\varphi \circ \sigma_{A_{0}}^{l}(\gamma)\right)$. But since $\varphi(K)=K$, we get $(*)$.

For $(* *)$, first note that since $I, J \in S\left(A_{K}\right)$, by the claim above, $u_{1}, u_{2}$ are monomials which do not contain negative powers of $\bar{x}_{n}$. Since $u \in \mathscr{M}$, we get that both $u_{1}, u_{2} \in \mathscr{M}$.

The other SE equations follow similarly. That $\left(R_{K}, S_{K}\right)$ is an $\mathrm{SE}$ induced by $\varphi \circ \sigma_{A_{0}}^{l} \mid \Sigma_{A_{K}}$ follows from the definition of induced SE and the fact that $\left.(R, S)\right)$ is an SE induced by $\varphi \circ \sigma_{A_{0}}^{l}$.

Finally, we show that $R_{K} \bar{A}_{K}^{m}=\bar{A}_{K}^{m+l}$. First, observe that since $\bar{A}^{m} R=\bar{A}^{m+l}$ and $R_{K}$ is over $Z^{+}\left[\bar{x}_{1}^{ \pm}, \ldots, \bar{x}_{n-1}^{ \pm}\right]$, we get

$$
\bar{A}_{K}^{m} R_{K} \leq \bar{A}_{K}^{m+l} \text {. }
$$

Let $u$ be a monomial in $\left(\bar{A}_{K}^{m+l}\right)_{I J}$. Of course, $I, J \in S\left(A_{K}\right)$. Then, since $\bar{A}_{K}^{m+l} \leq \bar{A}^{m+l}=\bar{A}^{m} R$ there is a state $\bar{I} \in S(A)$ and monomials $u_{1}, u_{2}$ such that

$$
u=u_{1} u_{2}, \quad u_{1} \leq\left(\bar{A}^{m}\right)_{\bar{I}}, \quad u_{2} \leq R_{\bar{I} J}
$$

To show that $\bar{A}_{K}^{m+l} \leq \bar{A}_{K}^{m} R_{K}$, it suffices to show $(*)$ and (**) above. The argument for $(* *)$ is exactly as above. For $(*)$, let $\gamma$ be a cycle in $K$ such that $\underline{i}(\gamma)=J$ and $R \bar{A}^{l(\gamma)}=\bar{A}^{l(\gamma)+l}$. Then

$$
u \mathrm{wt}_{\bar{A}}(\gamma) \leq\left(\bar{A}^{m}\right)_{I, \bar{I}} R_{\bar{I} J}\left(\bar{A}^{l(\gamma)}\right)_{J J} \leq\left(\bar{A}^{m}\right)_{I, \bar{I}}\left(\bar{A}^{l(\gamma)+l}\right)_{\bar{I}, J} .
$$


So, there is a path, with weight in $\mathscr{M}$, that meets $I, \bar{I}, J$. Since $K$ is irreducible and $\bar{A}$ is $F$-adapted, this implies that there is a cycle with weight in $\mathscr{M}$ that meets state $\bar{I}$ and the component $K$. Thus, $\bar{I} \in S\left(A_{K}\right)$.

A consequence of (3.4) is that for an automorphism $\varphi$ the induced permutation on boundary components is completely determined by any $\mathrm{SE}(R, S)$ induced by $\varphi$. The following shows more: the induced permutation is really determined only by $R$.

(3.10) Theorem. The permutations induced by an automorphism $\varphi$ on

(1) the cyclically moving subsets and

(2) the boundary components

are completely determined by $\Pi(\varphi)$ (where $\Pi$ is the dimension representation). Proof. (1) Use (3.8) and the fact that the map from automorphisms of $\Sigma_{A}$ to the group of permutations of the cyclically moving subsets is a homomorphism.

(2) Use (3.9) and the fact that the dimension representation is a homomorphism.

\section{SWITCHING CYCLES}

In this section, we ask when two cycles $\gamma, \eta \in \mathscr{C}$ can be switched by an automorphism. In the SFT case, Boyle, Lind and Rudolph [BLR, Theorem 7.2] showed that one can always switch cycles, in different orbits, of the same least period $=$ length provided that the cycles are sufficiently long. In the Markov chain setting, there is another necessary condition: the cycles must have the same wps. And, from $\S 3$, we have a significant necessary condition involving boundary components. In particular, in example (3.2), the two cycles of length 1 and weight 1 cannot be switched because their boundary components (which in this case happens to coincide with the cycles themselves) cannot be switched. Here, with these additional conditions, we extend the [BLR] result to the Markov chain setting.

Recent work of Kim and Roush [KR] indicated that there probably are some obstructions to switching cycles of "short" length in the SFT setting. Jack Wagoner now has an explicit example. See [KRW].

By an involution we mean an automorphism that is also an involution, as a map.

(4.1) Lemma.

(1) Let $\gamma, \eta \in \mathscr{C}$ with

$$
\begin{gathered}
l(\gamma)=l(\eta), \quad \operatorname{wps}(\gamma)=\operatorname{wps}(\eta), \\
\gamma, \eta \text { are in distinct orbits, }
\end{gathered}
$$

and

$$
\underline{t}\left(\sigma^{i} \gamma\right)=\underline{t}\left(\sigma^{i} \eta\right) \text { for some } i \text {. }
$$

Then, $\gamma$ and $\eta$ can be switched by an inert involution that fixes all cycles $\alpha \in \mathscr{C}$ with $l(\alpha) \leq l(\gamma)$ and $\alpha$ not in the orbit of $\gamma$ or $\eta$.

(2) Let $\gamma, \bar{\gamma}, \eta \in \mathscr{C}$ be cycles such that both pairs $\gamma, \bar{\gamma}$ and $\bar{\gamma}, \eta$ satisfy the hypothesis of (1). Then there is a composition, $\varphi$, of inert involutions such that $\varphi(\gamma)=\eta$ and that fixes all cycles $\alpha \in \mathscr{C}$ with $l(\alpha) \leq l(\gamma)$ and $\alpha$ not in the orbit of $\gamma, \eta$, or $\bar{\gamma}$. 
(3) Let $\gamma, \bar{\gamma}, \eta \in \mathscr{C}$ be cycles such that all three pairs satisfy the hypothesis of (1). Then there is a composition of inert involutions that switches $\gamma$ and $\eta$ and fixes all cycles $\alpha \in \mathscr{C}$ such that $l(\alpha) \leq l(\gamma)$ and $\alpha$ not in the orbit of $\gamma$ or $\eta$.

Note how the hypothesis and conclusion of (2) above are both weaker than the hypothesis and conclusion of (3) above.

Proof. The proof is straightforward, given [BLR, Theorem 7.2]. We give the ideas for completeness.

(1) We apply the frame code construction of [BLR, Theorem 7.2] and check that it preserves the weights of cycles and therefore defines an automorphism, in particular an involution, of $\Sigma_{A}$. We briefly review this construction.

The idea is that $\gamma$ and $\eta$ can be interchanged since they meet at the same state in the same phase. For a precise description, first, it is shown in the proof of [BLR, Theorem 7.2] that, by shifting $\gamma$ and $\eta$ if necessary, we may assume that $\gamma \eta \in \mathscr{C}$ i.e., the least period of $\gamma \eta$ is $2 l$, where $l=l(\gamma)$. Then the frame code is the automorphism $\varphi$ defined as follows: for $x \in \Sigma_{A}$ and $i \in \mathbf{Z}$, if $x_{i-2 l} \cdots x_{i+3 l-1}$ is a concatenation of five copies of $\gamma$ and $\eta$ (e.g., $\gamma \eta \gamma \gamma \eta$ ), then

$$
\varphi(x)_{i} \cdots \varphi(x)_{i+l-1}= \begin{cases}\eta & \text { if } x_{i} \cdots x_{i+l-1}=\gamma, \\ \gamma & \text { if } x_{i} \cdots x_{i+l-1}=\eta,\end{cases}
$$

and otherwise $\varphi(x)_{i}=x_{i}$. In the proof of [BLR, Theorem 7.2], it is shown that the frame code is well defined-roughly because $\gamma \eta \in \mathscr{C}$. It also fixes each of the desired cycles $\alpha$. The frame code is clearly a conjugacy and is an automorphism of the Markov chain $\Sigma_{A}$ (i.e., is measure-preserving) because $\gamma$ and $\eta$ have the same weight.

We claim that a frame code is a simple automorphism i.e., block isomorphic to some $c(I, \bar{A})$ and is therefore inert. The proof of this is a modification of Boyle's proof of inertness in the topological setting [B, Appendix]; we outline this in Appendix B.

(2) Apply (1) to the pairs $\gamma, \bar{\gamma}$ and $\bar{\gamma}, \eta$ to obtain involutions $\varphi_{1}, \varphi_{2}$. Then, $\varphi_{2} \circ \varphi_{1}$ is the desired automorphism.

(3) Again, apply (1) to the pairs $\gamma, \bar{\gamma}$ and $\bar{\gamma}, \eta$. to obtain involutions $\varphi_{1}$, $\varphi_{2}$. Then, $\varphi_{1} \circ \varphi_{2} \circ \varphi_{1}$ is the desired automorphism.

The following is an analogue of [BLR, Theorem 7.2] for Markov chains.

(4.2) Theorem. For each $w \in \mathrm{WPS}_{A}$, there exists $N_{w} \in \mathbf{N}$ such that if $\gamma, \eta$ are cycles satisfying

(I) $l(\gamma)=l(\eta) \geq N_{w}$.

(II) $\gamma, \eta \in \mathscr{C}_{w}$.

(III) Either $\gamma, \eta$ are in distinct orbits or $\eta=\sigma^{l(\gamma) / 2}(\gamma)$ or $\eta=\gamma$.

(IV) $K_{\gamma}$ and $K_{\eta}$ can be switched by an automorphism $\bar{\varphi}$ such that $\underline{i}(\bar{\varphi}(\gamma))$, $\underline{i}(\eta)$ (respectively, $\underline{i}(\bar{\varphi}(\eta)), \underline{i}(\gamma))$ lie in the same cyclic subset of $K_{\eta}$ (respectively, $\left.K_{\gamma}\right)$.

Then $\gamma$ and $\eta$ can be switched by an automorphism.

Moreover, $N_{w}$ is uniform over any closed subset of int $\overline{\mathrm{WPS}}_{A}$.

The conditions are necessary except for the largeness requirement on $l(\gamma)=$ $l(\eta)$. 
Remark. While condition (IV) is hard to check (the hard part is checking that $K_{\gamma}, K_{\eta}$ can be switched), it is vacuous if $\operatorname{wps}(\gamma)=\operatorname{wps}(\eta) \in \operatorname{int} \overline{\mathrm{WPS}}_{A}$ or if each $A_{F}$ is mixing (e.g., if $A$ is Bernoulli). Also, if we require the automorphism that switches $\gamma, \eta$ to be inert, then by (3.8) and (3.9), we must have $K_{\gamma}=K_{\eta}$ and $\underline{i}(\gamma), \underline{i}(\eta)$ must be in the same cyclic subset. Under this assumption, and the assumption that $\gamma, \eta$ are in distinct orbits, the automorphism produced by (4.2) is indeed inert-see (4.3) below.

Proof of (4.2). The necessity is clear. We first prove a stronger version of sufficiency in a special case:

(4.3) Lemma. For $w \in \mathbf{W P S}_{A}$, there exists $N_{w} \in \mathbf{N}$ such that if $\gamma, \eta$ are cycles satisfying I, II above and

(III' $\left.{ }^{\prime}\right) \gamma, \eta$ are in distinct orbits,

$\left(\mathrm{IV}^{\prime}\right) K_{\gamma}=K_{\eta}$ and $\underline{i}(\gamma), \underline{i}(\eta)$ are in the same cyclic subset,

then $\gamma, \eta$ can be switched by a composition of inert involutions which fixes all other cycles $\alpha \in \mathscr{C}$ with $l(\alpha) \leq l(\gamma)$ and $\alpha$ not in the same orbit as $\gamma$ or $\eta$.

Proof of (4.3). For each boundary component $K$, there is an integer $L$ such that for each pair of states $I, J$ in the same cyclic subset of $K$, there is a path $\alpha_{I, J}^{K}$ in $K$ from $I$ to $J$ with length $L$.

Now, for $w \in \mathrm{WPS}_{A}$, we can apply (1.1)(2) to each of the boundary components $K \in S(w)$, paths $\alpha=\alpha_{I, J}^{K}, W=\{w\}$, and $M=3$; let

$$
N_{w}=\max _{\substack{K \in S(w) \\ I, J}} N_{A_{K}}\left(\alpha_{I, J}^{K},\{w\}, 3\right)
$$

Let $\gamma, \eta$ satisfy (I), (II), (III'), (IV'). Write $\gamma=e_{1} \cdots e_{l(\gamma)}$ and let $I=$ $\underline{t}\left(e_{l(\gamma)-L}\right), J=\underline{t}(\eta)$. Then, there is a cycle $\bar{\gamma} \in \mathscr{C}_{w}\left(A_{K_{\gamma}}\right)$ such that

$$
l(\bar{\gamma})=l(\gamma)=l(\eta)
$$

and $\bar{\gamma}$ has suffix $\alpha_{I, J}^{K}$. Since $M=3$ above, we may assume that $\bar{\gamma}$ is not in the orbit of $\gamma$ or $\eta$. Thus, we can apply (4.1)(3). This gives (4.3)

Now back to the proof of (4.2).

Let $\gamma, \eta$ satisfy (I), (II), (III), (IV).

If $\eta=\sigma^{l(\gamma) / 2}(\gamma)$, then $\varphi=\sigma^{l(\gamma) / 2}$ will do. So, we may assume that $\gamma, \eta$ are in distinct orbits. If $K_{\gamma}=K_{\eta}$, then either $\underline{i}(\gamma), \underline{i}(\eta)$ are in the same cyclic subset of $K_{\gamma}$ or $d_{A_{K_{\gamma}}}$ is even and $i\left(\sigma_{A_{0}}^{\left(d_{A_{\gamma}} / 2\right)}(\gamma)\right), \underline{i}(\eta)$ lie in the same cyclic subset of $K_{\gamma}=K_{\eta}$. The former case was the special case in (4.3) above. For the latter case, again by (4.3), there is an automorphism which switches $\sigma_{A_{0}}^{\left(d_{A_{K_{y}}} / 2\right)}(\gamma)$ and $\eta$. Now, compose this automorphism with $\sigma_{A_{0}}^{\left(d_{A_{K_{y}}} / 2\right)}$.

So, we may assume $K_{\gamma} \neq K_{\eta}$. Let $\bar{\varphi}$ be the automorphism in IV. We claim that there is an automorphism $\varphi_{1}$ such that $\varphi_{1}(\bar{\varphi}(\eta))=\gamma$ and $\varphi_{1}$ fixes $\bar{\varphi}(\gamma)$, $\eta$. This comes from (4.3) if $\bar{\varphi}(\eta)$ and $\gamma$ are in distinct orbits. If $\bar{\varphi}(\eta)$ and $\gamma$ are in the same orbit, then again, as in the proof of (4.3), we can find a cycle $\bar{\gamma} \in C_{w}^{A_{K_{\gamma}}}$ such that $l(\bar{\gamma})=l(\gamma), \bar{\gamma}$ not in the orbit of $\gamma$ and $\bar{\gamma}$ has $\alpha_{I, J}^{K_{\gamma}}$ as a suffix where $J=\underline{t}(\bar{\varphi}(\eta))$ and $I=\underline{t}\left(e_{l(\gamma)-L}\right), \gamma=e_{1} \cdots e_{l(\gamma)}$. Now 4.1(2) gives us our desired automorphism $\varphi_{1}$. 
Likewise, there is an automorphism $\varphi_{2}$ such that $\varphi_{2}(\bar{\varphi}(\gamma))=\eta$ and $\varphi_{2}$ fixes $\bar{\varphi}(\eta), \gamma$. Now, set $\varphi=\varphi_{2} \circ \varphi_{1} \circ \bar{\varphi}$.

The uniformity of $N_{w}$ follows from the uniformity in (1.1)(2).

\section{(4.4) Corollary.}

(1) If $\Sigma_{A}$ is Bernoulli, then in (4.2), each $N_{w}$ can be chosen to be 1.

(2) If each $A_{F}$ is irreducible, then in (4.2) $N_{w}$ can be chosen uniform on $\overline{\mathrm{WPS}}_{A}=\mathrm{WPS}_{A}$.

Proof. (1) Since a Bernoulli shift has only one state, we can apply (4.1)(1) immediately. For the proof of (2), we need

\section{(4.5) Proposition.}

(1) Let $F$ be a face of $\overline{\mathrm{WPS}}_{A}$. There is an open neighborhood (in $\overline{\mathrm{WPS}}_{A}$ ) $B_{F}$ of $F$ such that for all $w \in B_{F}$ and all cycles $\gamma=e_{1} \cdots e_{l(\gamma)} \in \mathscr{C}_{w}$,

$$
\frac{\#\left\{i: \underline{t}\left(e_{i}\right) \in S\left(A_{F}\right)\right\}}{l(\gamma)}>\frac{1}{2} .
$$

(2) Let $F$ be a face such that $A_{F}$ is irreducible. Let $W_{F}$ be a closed subset of int $F$. Let $\alpha$ be a path in $G\left(A_{F}\right)$. There exists $M=M\left(\alpha, W_{F}\right)$ and an open neighborhood (in $\overline{\mathrm{WPS}}_{A}$ ) $W_{F}^{\prime}$ of $W_{F}$ such that for all $w \in W_{F}^{\prime}$

$$
\mathscr{L}\left(\mathscr{C}_{w}\right) \cap[M, \infty)=\mathscr{L}\left(\mathscr{C}_{w, \alpha}\right) \cap[M, \infty) .
$$

Moreover, for $l \in \mathscr{L}\left(\mathscr{C}_{w}\right) \cap[M, \infty)$, there are at least three cycles in $\mathscr{C}_{w, \alpha}$ in pairwise distinct orbits, with length equal to $l$.

Before we prove (4.5), we show how to prove (4.4)(2), given (4.5). We assume, only for simplicity, that each $A_{F}$ is mixing. Note that, in this case, condition (IV) is vacuous. We may assume that condition (III) is replaced by $\left(\mathrm{III}^{\prime}\right)$, i.e., $\gamma, \eta$ are in distinct orbits. We show inductively that for each positive $m$, there exists $N_{m}$ and a neighborhood (in $\overline{\mathrm{WPS}}_{A}$ ) $U_{m}$ of $\bigcup_{\{m \text {-faces } F\}} F$ such that if $\gamma, \eta$ are cycles with $l(\gamma)=l(\eta) \geq N_{m}, \gamma, \eta \in \mathscr{C}_{w}, w \in U_{m}$ and $\gamma, \eta$ are in distinct orbits, then $\gamma$ and $\eta$ can be switched by an automorphism, (the case $m=$ dimension $\overline{\mathrm{WPS}}_{A}$ yields (4.4)(2)).

For each face $F$ and states $I, J$ in $G_{F}$, we can choose paths $\alpha_{I, J}^{F}$ in $G_{F}$, all of the same length $L$, with $\underline{i}\left(\alpha_{I, J}^{F}\right)=I$ and $\underline{t}\left(\alpha_{I, J}^{F}\right)=J$. Now, assume $N_{m}, U_{m}$ have been found as above, with $m \geq-1$. Let $F$ be an $(m+1)$-face. Then, $W_{F} \equiv F-U_{m}$ is a closed subset of int $F$. Apply (4.5)(2). Let

$$
N^{F}=\max _{I, J} M\left(\alpha_{I, J}^{F}, W_{F}\right) .
$$

If $w \in B_{F}$ and $\gamma=e_{1} \cdots e_{l(\gamma)}, \eta=f_{1} \cdots f_{l(\eta)}$ are cycles such that $l(\gamma)=l(\eta)$ and $\gamma, \eta \in \mathscr{C}_{w}$, then by (4.5)(1), for some $i, \underline{t}\left(e_{i}\right), \underline{t}\left(f_{i+L}\right) \in S\left(A_{F}\right)$. If also $w \in W_{F}^{\prime}, l(\gamma)=l(\eta) \geq N^{F}$, and $\gamma, \eta$ are in distinct orbits, then by (4.5)(2), there is a cycle $\bar{\gamma}=\bar{e}_{1} \cdots \bar{e}_{l(\bar{\gamma})} \in \mathscr{C}_{w}(A)$ with $l(\bar{\gamma})=l(\gamma)=l(\eta)$ such that $\underline{t}\left(\bar{e}_{i}\right)=\underline{t}\left(e_{i}\right), \underline{t}\left(\bar{e}_{i+L}\right)=\underline{t}\left(f_{i+L}\right)$ and $\bar{\gamma}, \gamma, \eta$ in distinct orbits. Now apply (4.1)(3) to switch $\gamma$ and $\eta$.

Let

$$
N_{m+1}=\max \left(N_{m}, \max _{\{(m+1) \text {-faces } F\}} N^{F}\right) .
$$


Let

$$
U_{m+1}=U_{m} \cup\left(\bigcup_{\{(m+1) \text {-faces } F\}}\left(W_{F}^{\prime} \cap B_{F}\right)\right),
$$

an open neighborhood of the union of all $(m+1)$-faces. Then, $N_{m+1}, U_{m+1}$ satisfy the above.

So, it remains to prove (4.5). We need the following lemmas.

(4.6) Lemma. Let $\eta_{1}, \ldots, \eta_{k}$ be cycles such that $w=\sum_{i}\left(l_{i} / \sum_{j} l_{j}\right) \operatorname{wps}\left(\eta_{i}\right) \in$ int $\overline{\mathrm{WPS}}_{A}$, (where $\left.l_{i}=l\left(\eta_{i}\right)\right)$. Then $d(w)$ divides $\sum_{i} l_{i}$.

Proof. We may assume $A$ is a $\Delta$-form. Clearly $d_{A} \mid \sum_{i} l_{i}$. And

$$
\left(\sum_{i} l_{i}\right) w=\sum_{i} \log \mathrm{wt}\left(\eta_{i}\right) \in \log \Delta_{A} .
$$

(4.7) Lemma. Let $\gamma$ be a cycle and let $F$ be a face such that $\gamma$ meets $G\left(A_{F}\right)$. Then $(\gamma)$ is equivalent to a sequence $S=\left(c_{1}, \ldots, c_{n}\right)$ of cycles such that for some $m$ :

(1) For all $1 \leq i \leq m, c_{i}$ is simple and $\operatorname{wps}\left(c_{i}\right) \in F$.

(2) For $m<\bar{i} \leq \bar{n}, c_{i}$ meets $G\left(A_{F}\right)$ and the singleton $\left(c_{i}\right)$ is equivalent to a sequence of simple cycles, each of whose wps does not belong to $F$.

Proof. $(\gamma)$ is equivalent to a sequence of simple cycles $S=\left(s_{1}, \ldots, s_{k}\right)$. Let $F_{0}=\left\{i \in\{1, \ldots, k\}: s_{i}\right.$ lies in $\left.G\left(A_{F}\right)\right\}$. Let $S_{0}=S\left(G\left(A_{F}\right)\right)$. Inductively, define $F_{j}=\left\{i \in\{1, \ldots, k\}: i \notin \bigcup_{l=0}^{j-1} F_{l}\right.$ but $s_{i}$ meets $\left.S_{j-1}\right\}$. Let $S_{j}=\bigcup_{i \in F_{j}}$ the states that $s_{i}$ meets $\}$.

Since $\gamma$ is a cycle which meets $G\left(A_{F}\right)$, for some $J,\left\{F_{j}: 0 \leq j \leq J\right\}$ partition $\{1, \ldots, k\}$. For each $j \geq 2$ and $i \in F_{j}$, there exists $i^{\prime} \in F_{j-1}$ such that $s_{i}$ meets $s_{i^{\prime}}$. Let $\Phi(i)=i^{\prime}$ (note that $i^{\prime}$ need not be unique, but for each $i$, we just pick one such $i^{\prime}$ ). For each $i \in F_{1}$, there is a cycle $\gamma^{(i)}$ such that the singleton $\left(\gamma^{(i)}\right)$ is equivalent to

$$
\left(s_{k}: k \in\{i\} \cup \Phi^{-1}(i) \cup \Phi^{-2}(i) \cup \cdots \cup \Phi^{-(J-1)}(i)\right) .
$$

Let $m=\left|F_{0}\right|, n=\left|F_{0} \cup F_{1}\right|$. Let $\Psi:\{1, \ldots, n\} \rightarrow F_{0} \cup F_{1}$ be a bijection such that $\Psi(\{1, \ldots, m\})=F_{0}$. Define

$$
c_{i}= \begin{cases}S_{\Psi(i)}, & 1 \leq i \leq m, \\ \gamma^{(\Psi(i))}, & m<i \leq n .\end{cases}
$$

Recall that $\left\langle\mathrm{WPS}_{A}\right\rangle_{Q}$ inherits a metric, once it is identified with $Q^{n}$. For a set $V$, and $\delta>0$, we let

$$
B_{\delta}(V)=\{x: d(x, F)<\delta\}, \quad \bar{B}_{\delta}(V)=\{x: d(x, F) \leq \delta\} .
$$

(4.8) Lemma. Let $F$ be a face of a rational polytope $P$. Let $G$ be a closed subset of $P$ such that $F \cap G=\varnothing$. Then, $\forall \varepsilon>0 \quad \exists \delta=\delta(\varepsilon)>0$ such that if $x \in F, y \in \bar{B}_{\delta}(F), z \in G$ and $x, y, z$ are collinear, then $d(x, y)<\varepsilon$.

Proof. We may assume $P$ is a real polytope by replacing it by its closure in $\mathbf{R}^{n}$. We argue by contradiction. If (4.8) were false, then for some $\varepsilon>0$, there would exist $x_{n} \in F, y_{n} \in \bar{B}_{1 / n}(F), z_{n} \in G$, with $x_{n}, y_{n}, z_{n}$ collinear, and $d\left(x_{n}, y_{n}\right) \geq \varepsilon$. By compactness, there exist $x \in F, y \in F, z \in G$ such 
that $x, y, z$ are collinear and $d(x, y) \geq \varepsilon$. But any line which meets $F$ and contains a point in $P-F$ can meet $F$ in only one point. Thus, $x=y$, a contradiction.

Proof of (4.5). Let $\widetilde{F}$ be the (rational) convex hull of $\{\operatorname{wps}(\gamma): \gamma$ is a simple cycle and $\operatorname{wps}(\gamma) \notin F\}$. Then, $\widetilde{F}$ is closed and disjoint from $F$. Now, any cycle $\gamma$ is equivalent to a sequence $S=\left(s_{1}, \ldots, s_{m}, s_{m+1}, \ldots, s_{n}\right)$ of simple cycles, with $\operatorname{wps}\left(s_{i}\right) \in F, i=1, \ldots, m$, and $\operatorname{wps}\left(s_{i}\right) \notin F, i=m+1, \ldots, n$.

Let

$$
\begin{gathered}
w=\operatorname{wps}(\gamma), \quad t=\frac{\sum_{i=1}^{m} l\left(s_{i}\right)}{l(\gamma)}, \\
w_{F}=\sum_{i=1}^{m} \frac{l\left(s_{i}\right)}{t l(\gamma)} \operatorname{wps}\left(s_{i}\right) \in F, \quad \tilde{w}_{F}=\sum_{i=m+1}^{n} \frac{l\left(s_{i}\right)}{(1-t) t(\gamma)} \operatorname{wps}\left(s_{i}\right) \in \widetilde{F} .
\end{gathered}
$$

Note that

$$
w=t w_{F}+(1-t) \tilde{w}_{F} .
$$

So,

and so

$$
d\left(w, w_{F}\right)=(1-t) d\left(w_{F}, \tilde{w}_{F}\right) \geq(1-t) d(F, \widetilde{F})
$$

$$
t \geq 1-\frac{d\left(w, w_{F}\right)}{d(F, \widetilde{F})} .
$$

So, if $d\left(w, w_{F}\right)<d(F, \tilde{F}) / 2$, then $t>\frac{1}{2}$. Choose $\delta=\delta(d(F, \widetilde{F}) / 2)$, as in (4.8), and let $B_{F}=B_{\delta}(F)$.

For part (2), we may assume that $G\left(A_{F}\right)$ does not consist entirely of a single cycle. Let $W_{F}$ be a closed of int $F$. Let

$$
\varepsilon<\min \left(\frac{d\left(W_{F}, \partial F\right.}{2}, \frac{d(F, \tilde{F})}{3}\right) .
$$

Let $\delta=\min (\varepsilon, \delta(\varepsilon))$ where $\delta(\varepsilon)$ is as in (4.8) applied to $G=\widetilde{F}$ in the polytope $\overline{\mathrm{WPS}}_{A}$. Let $W_{F}^{\prime}=B_{\delta}\left(W_{F}\right)$, let $\alpha$ be a path in $G\left(A_{F}\right)$, let $\alpha^{\prime \prime}$ be a cycle in $G\left(A_{F}\right)$, which meets every state of $G\left(A_{F}\right)$ and satisfies $\underline{t}\left(\alpha^{\prime \prime}\right)=\underline{i}(\alpha)$, and let $\alpha^{\prime}=\alpha^{\prime \prime} \alpha$.

Apply $(1.1)(2)$ to get $N_{A_{F}}\left(\alpha^{\prime}, \bar{B}_{2 \varepsilon}\left(W_{F}\right) \cap F, 3\right)$ with the properties as in $(1.1)(2)$. Note that by the choice of $\varepsilon, W=\bar{B}_{2 \varepsilon}\left(W_{F}\right) \cap F$ is a closed subset of int $F$.

Our desired $M\left(\alpha, W_{F}\right)$ is

$$
M=M\left(\alpha, W_{F}\right)=\max \left(6 l\left(\alpha^{\prime}\right), 2 N_{A_{F}}\left(\alpha^{\prime}, \bar{B}_{2 \varepsilon}\left(W_{F}\right) \cap F, 3\right)\right) .
$$

We must show that for $w \in W_{F}^{\prime}$, if $\gamma \in \mathscr{C}_{w}$ and $l(\gamma) \geq M$, then there are at least three cycles $\bar{\gamma} \in \mathscr{C}_{w, \alpha}$ in distinct orbits with $l(\bar{\gamma})=l(\gamma)$. Note that since $W_{F}^{\prime} \subset B_{F}$ (as constructed in part 1), $\gamma$ must meet $G\left(A_{F}\right)$. So, we can apply (4.7) to get $(\gamma)$ equivalent to a sequence $\left(c_{1}, \ldots, c_{n}\right)$ of cycles such that for $i=1, \ldots, m, c_{i}$ is simple and $\operatorname{wps}\left(c_{i}\right) \in F$, and for $i=m+1, \ldots, n$, $c_{i}$ meets $G\left(A_{F}\right)$ and $\left(c_{i}\right)$ is equivalent to a sequence of simple cycles, each of whose wps belongs to $\widetilde{F}$. 
Let

$$
\begin{gathered}
w_{F}=\sum_{i=1}^{m} \frac{l\left(c_{i}\right)}{\sum_{j=1}^{m} l\left(c_{j}\right)} \operatorname{wps}\left(c_{i}\right) \in F, \\
\tilde{w}_{F}=\sum_{i=m+1}^{n} \frac{l\left(c_{i}\right)}{\sum_{j=m+1}^{n} l\left(c_{j}\right)} \operatorname{wps}\left(c_{i}\right) \in \tilde{F} .
\end{gathered}
$$

Note that

$$
w=t w_{F}+(1-t) \tilde{w}_{F}, \quad t=\frac{\sum_{i=1}^{m} l\left(c_{i}\right)}{l(\gamma)} .
$$

Case 1. $w=w_{F}$

Then, $w \in W_{F}^{\prime} \cap F$. And

$$
l(\gamma) \in \mathscr{L}\left(\mathscr{C}_{w}\left(A_{F}\right)\right) \cap\left[N_{A_{F}}\left(\alpha^{\prime}, \bar{B}_{2 \varepsilon}\left(W_{F}\right) \cap F, 3\right), \infty\right) .
$$

Thus, by $(1.1)(2)$, there are at least three cycles $\bar{\gamma} \in \mathscr{C}_{w, \alpha^{\prime}}\left(A_{F}\right) \subset \mathscr{C}_{\omega, \alpha}(A)$, in distinct orbits, with $l(\bar{\gamma})=l(\gamma)$.

Case 2. $w \neq w_{F}$.

Since $w_{F} \in F, w \in \bar{B}_{\delta}(F)$ and $\tilde{w}_{F} \in \widetilde{F}$ and since, by (7), $w_{F}, w, \tilde{w}_{F}$ are collinear, we get, by the choice of $\delta$ and $(4.8), d\left(w_{F}, w\right)<\varepsilon$. Thus, $d\left(w_{F}, W_{F}\right)<\varepsilon+\delta \leq 2 \varepsilon$. So,

$$
w_{F} \in B_{2 \varepsilon}\left(W_{F}\right) \cap F \subset \text { int } F .
$$

Now, with $t$ as in (7) above,

$$
\varepsilon>d\left(w_{F}, w\right)=(1-t) d\left(w_{F}, \tilde{w}_{F}\right)>(1-t) 3 \varepsilon
$$

(the latter inequality by the choice of $\varepsilon$ ). Thus,

$$
t>\frac{2}{3} \text {. }
$$

So,

$$
\sum_{i=1}^{m} l\left(c_{i}\right)>\frac{2}{3} l(\gamma)>N_{A_{F}}\left(\alpha^{\prime}, \bar{B}_{2 \varepsilon}\left(W_{F}\right) \cap F, 3\right),
$$

the latter by the choice of $M$. Also, by (8) above and (4.6), applied to $A_{F}$,

$$
d_{A_{F}}\left(w_{F}\right) \text { divides } \sum_{i=1}^{m} l\left(c_{i}\right) \text {. }
$$

Thus, by (1.1), there are three cycles $\gamma^{\prime} \in \mathscr{C}_{w_{F}, \alpha^{\prime}}\left(A_{F}\right)$ in distinct orbits such that $l\left(\gamma^{\prime}\right)=\sum_{i=1}^{m} l\left(c_{i}\right)$. Fix one such $\gamma^{\prime}$. We may write $\gamma^{\prime}=\gamma^{\prime \prime} \alpha^{\prime}=\gamma^{\prime \prime} \alpha^{\prime \prime} \alpha$. Since $\alpha^{\prime \prime}$ meets each state of $G\left(A_{F}\right)$, after reordering $c_{m+1} \cdots c_{n}$ if necessary, we may decompose $\alpha^{\prime \prime}=\alpha_{m+1} \cdots \alpha_{n}$ such that $c_{m+1} \alpha_{m+1} \ldots c_{n} \alpha_{n}$ is an allowed cycle. Let

$$
\bar{\gamma}=\gamma^{\prime \prime} c_{m+1} \alpha_{m+1} \cdots c_{n} \alpha_{n} \alpha
$$


Then,

$$
\begin{aligned}
l(\bar{\gamma}) & =l\left(\gamma^{\prime \prime}\right)+\sum_{i=m+1}^{n} l\left(c_{i}\right)+\sum_{i=m+1}^{n} l\left(\alpha_{i}\right)+l(\alpha) \\
& =l\left(\gamma^{\prime}\right)+\sum_{i=m+1}^{n} l\left(c_{i}\right)=\sum_{i=1}^{n} l\left(c_{i}\right)=l(\gamma)
\end{aligned}
$$

and

$$
\begin{aligned}
\mathrm{wt}(\bar{\gamma}) & =\mathrm{wt}\left(\gamma^{\prime \prime}\right)\left(\prod_{i=m+1}^{n} \mathrm{wt}\left(c_{i}\right)\right)\left(\prod_{i=m+1}^{n} \mathrm{wt}\left(\alpha_{i}\right)\right) \mathrm{wt}(\alpha) \\
& =\mathrm{wt}\left(\gamma^{\prime}\right) \cdot \prod_{i=m+1}^{n} \mathrm{wt}\left(c_{i}\right)=\prod_{i=1}^{n} \mathrm{wt}\left(c_{i}\right)=\mathrm{wt}(\gamma) .
\end{aligned}
$$

Clearly, $\bar{\gamma}$ has $\alpha$ as a suffix. So, $l(\bar{\gamma})=l(\gamma)$ and $\bar{\gamma} \in \mathscr{C}_{w, \alpha}$ provided that $\bar{\gamma} \in \mathscr{C}$, i.e., $\gamma$ has least period $l(\gamma)$. Well, by $(9)$,

$$
(2 t-1) l(\gamma)>\frac{1}{3} l(\gamma)
$$

By the choice of $M($ recall $l(\gamma) \geq M), \frac{1}{3} l(\gamma)>2 l\left(\alpha^{\prime}\right)$. Thus,

$$
(2 t-1) l(\gamma)>2 l\left(\alpha^{\prime}\right)
$$

Recalling that $\gamma^{\prime}=\gamma^{\prime \prime} \alpha^{\prime}$,

$$
l\left(\gamma^{\prime \prime}\right)=l\left(\gamma^{\prime}\right)-l\left(\alpha^{\prime}\right)=\left(\sum_{i=1}^{m} l\left(c_{i}\right)\right)-l\left(\alpha^{\prime}\right)=t l(\gamma)-l\left(\alpha^{\prime}\right)
$$

This, together with (10), gives

$$
l\left(\gamma^{\prime \prime}\right)>(1-t) l(\gamma)+l\left(\alpha^{\prime}\right)=\left(\sum_{i=m+1}^{n} l\left(c_{i}\right)\right)+l\left(\alpha^{\prime}\right) .
$$

So, $l\left(\gamma^{\prime \prime}\right)>\frac{1}{2} l(\bar{\gamma})$. But if $\bar{\gamma}$ has least period $<l(\bar{\gamma})$, then it has least period $\leq \frac{1}{2} l(\bar{\gamma})$ and thus $c_{n}$ would appear in $\gamma^{\prime \prime}$. But $\gamma^{\prime \prime}$ lies in $G\left(A_{F}\right)$ and $c_{n}$ does not. Thus, $\bar{\gamma}$ has least period $=l(\bar{\gamma})$. So, we have produced one of the desired cycles in $\mathscr{C}_{w, \alpha}$. We actually get three such cycles in distinct orbits since the construction of $\bar{\gamma}$ from distinct cycles $\gamma^{\prime}$ yields three cycles $\bar{\gamma}$ in distinct orbits (for this, use the fact that the cycles $c_{i}, i=m+1 \ldots n$, must all leave $G_{F}$ ).

\section{REALIZING PERMUTATIONS OF CYCLES BY COMPOSITIONS OF INVOLUTIONS: BERNOULLI SHIFTS}

In this section, we focus on Bernoulli shifts and, in this case, we give a complete characterization of which permutations of finitely many cycles can be realized by a composition of involutions. This generalizes a result of Boyle and Krieger [BK, 3.10]. Ultimately there should be versions of this for general Markov chains, that incorporates the ideas of $\S \S 3$ and 4 , but that will have to wait until the theory of automorphisms of SFT's is more fully developed.

The conditions are given in terms of the Sign Gyration Compatibility Condition (SGCC), first formulated by Boyle and Krieger [BK], and defined as follows. 
For $n \in \mathbf{N}$ let $\mathscr{C}(n)$ be the set of all cycles $\gamma \in \mathscr{C}$ with $l(\gamma)=n$. Let $\mathscr{O}(n)$ be the set of $\sigma_{A_{0}}$-orbits of cycles $\gamma \in \mathscr{C}(n)$. Let $\mathscr{U}$ be a shift-invariant collection of cycles in $\mathscr{C}$. Let $\mathscr{O}$ be the set of $\sigma_{A_{0}}$-orbits of cycles $\gamma \in \mathscr{U}$. Let $\pi$ be a shift-commuting permutation of $\mathscr{U}$. Now, $\pi$ must preserve length and so $\pi$ induces permutations $\left.\pi\right|_{\mathscr{E}(n) \cap \mathscr{U}}$. Let $\rho_{n}(\pi)$ denote the induced permutation on the $\sigma_{A_{0}}$-orbits, $\mathscr{O}(n) \cap \mathscr{O}$. Let $g_{n}(\pi)$ denote the $n$th gyration number of $\pi$ : for each $\sigma_{A_{0}}$-orbit $\theta \in \mathscr{O}(n) \cap \mathscr{O}$, pick precisely one representative $\gamma_{\theta} \in \mathscr{C}(n) \cap \mathscr{U}$ of $\theta$ and define

$$
g_{n}(\pi) \equiv \sum_{\{\theta \in \mathscr{O}(n) \cap \mathcal{O}\}} s\left(\gamma_{\theta}\right) \bmod n
$$

where $s\left(\gamma_{\theta}\right)$ is defined by

$$
\pi\left(\gamma_{\theta}\right)=\sigma^{s\left(\gamma_{\theta}\right)} \gamma_{\rho_{n}(\pi)(\theta)}
$$

The Sign Gyration Compatibility Condition (SGCC) on $\pi$ is the following: whenever $m$ is a nonnegative integer and $q$ is an odd positive integer, then

$$
g_{2^{m} q}(\pi)=\left\{\begin{array}{l}
0 \bmod 2^{m} q \text { if } \prod_{j=1}^{m-1} \operatorname{sign} \rho_{2^{j} q}(\pi)=1, \\
2^{m-1} q \bmod 2^{m} q \text { if } \prod_{j=1}^{m-1} \operatorname{sign} \rho_{2^{j} q}(\pi)=-1 .
\end{array}\right.
$$

Note that the SGCC is preserved under composition.

Let $\mathscr{C}^{N}=\bigcup_{n=1}^{N} \mathscr{C}(n)$. We will show

(5.1) Theorem. Let $\Sigma_{A}$ be a Bernoulli shift and $N \in \mathbf{N}$. Let $\pi$ be a permutation of $\mathscr{C}^{N}$. Then, $\pi$ is realized by a composition of involutions iff $\pi$ is shift-commuting, weight-preserving and for each $\left.w \in \mathrm{WPS}_{A} \pi\right|_{\mathscr{C}^{N} \cap \mathscr{E}_{w}}$ satisfies the SGCC.

This generalizes Boyle-Krieger [BK, 3.10]. Note that since $\mathscr{C}^{N}$ is finite, there are only finitely many $w \in \mathrm{WPS}_{A}$ that occur.

For an automorphism $\varphi$ of $\Sigma_{A}$ and $w \in$ WPS $_{A}$ there are well-defined bijections $\varphi_{w}: \mathscr{C}_{w} \rightarrow \mathscr{C}_{w}$ obtained by restricting $\varphi$ to $\mathscr{C}_{w}$.

(5.2) Lemma. Let $\varphi$ be an involution of $\Sigma_{A}$. For each $w \in \operatorname{WPS}_{A}, \varphi_{w}$ satisfies the SGCC.

We defer the proof of this until the end.

Proof of (5.1). Only if: Let $\varphi$ be a composition of involutions such that for each $\gamma \in \mathscr{C}^{N} \varphi(\gamma)=\pi(\gamma)$. Then, clearly $\pi$ must be shift-preserving and weight-preserving. Apply (5.2).

If: Since $\pi$ is weight-preserving, $\pi\left(\mathscr{C}^{N} \cap \mathscr{C}_{w}\right)=\mathscr{C}^{N} \cap \mathscr{C}_{w}$ for each $w \in$ $\mathrm{WPS}_{A}$. Let $\pi_{w}$ be the permutation of $\mathscr{C}^{N}$ :

$$
\pi_{w}(\gamma)= \begin{cases}\pi(\gamma) & \text { if } \gamma \in \mathscr{C}_{w}, \\ \gamma & \text { otherwise. }\end{cases}
$$

Then $\pi_{w}$ satisfies the SGCC.

One shows (exactly as in [BK, Lemma 3.7]), that any shift-commuting, weightpreserving permutation $\bar{\pi}$ of $\mathscr{C}^{n+1}$ such that $\left.\bar{\pi}\right|_{\mathscr{C}_{n}}=$ identity and $g_{n+1}(\bar{\pi})=0$ can be realized by a composition of involutions. (The idea of the proof is to write $\bar{\pi}$ as a product of special transpositions and then apply (4.1)(1)). 
Now, arguing as in [BK, Theorem 3.8], we show inductively that $\left.\pi_{w}\right|_{\mathscr{E}_{n}}$ can be realized by a composition of involutions and thus $\pi_{w}=\left.\pi_{w}\right|_{\mathscr{C}_{N}}$ can be so realized. Assume the inductive hypothesis. Let $\varphi_{n}$ realize $\left.\pi_{w}\right|_{\mathscr{C}_{n}}, n<N$. Since $\pi_{w}$ satisfies the SGCC, and $\varphi_{n}$ satisfies the SGCC (by (5.2)), $\left.\pi_{w} \circ \varphi_{n}^{-1}\right|_{\mathscr{E} n+1}$ satisfies the SGCC. Thus, since $\left.\pi_{w} \circ \varphi_{n}^{-1}\right|_{\mathscr{C} n}=$ identity, we get $g_{n+1}\left(\left.\pi_{w} \circ \varphi_{n}^{-1}\right|_{\mathscr{C}_{n+1}}\right)=$ 0 . So, by the remarks in the previous paragraph, $\left.\bar{\pi} \equiv \pi_{w} \circ \varphi_{n}^{-1}\right|_{\mathscr{E}_{n+1}}$ can be realized by a composition $\psi_{n}$ of involutions. Set $\varphi_{n+1}=\psi_{n} \circ \varphi_{n}$. So, $\left.\pi_{w}\right|_{\mathscr{E} n+1}$ can be realized. So, $\pi_{w}$ can be realized. Now, compose the $\pi_{w}$ in any order.

It now remains to prove (5.2).

For the proof of (5.2), we need to recall the notion of zeta function. Let $T$ be a bijection of a set $X$ such that $N_{n} \equiv N_{n}^{T} \equiv \#\left\{x \in X: T^{n} x=x\right\}<\infty$. The zeta function of $T$ is defined:

$$
\zeta_{T}(s)=\exp \left(\sum_{n=1}^{\infty} \frac{N_{n}}{n} s^{n}\right), \quad s \in \mathbf{R} .
$$

For an SFT $\Sigma_{A_{0}}$, it is well known (see Bowen and Lanford [BL]) that

$$
\zeta_{\sigma_{A_{0}}}(s)=\frac{1}{\operatorname{det}\left(I-s A_{0}\right)}
$$

Clearly, the zeta function is invariant under conjugacy of bijections.

Let $A$ be a stochastic form (i.e., $A_{1}$ is a stochastic matrix). Define the stochastic zeta function, associated to a Markov chain $\Sigma_{A}$,

$$
\zeta_{A}(t, s) \equiv \exp \left(\sum_{n=1}^{\infty} \frac{\operatorname{Trace}\left(A_{t}\right)^{n}}{n} s^{n}\right), \quad t, s \in \mathbf{R} .
$$

Parry and Williams $[\mathrm{PW}]$ noted that

$$
\zeta_{A}(t, s)=\frac{1}{\operatorname{det}\left(I-s A_{t}\right)}
$$

Note that we have suppressed the role of the shift. Clearly, the stochastic zeta function is an invariant of block isomorphism. Also, $\zeta_{A}=\zeta_{B}$ iff for every $w \in \mathrm{WPS}_{A} \zeta_{\left(\sigma_{A_{0}}\right)_{w}}=\zeta_{\left(\sigma_{B_{0}}\right)_{w}}$.

The next result, from [BK, Lemma (3.3)], was stated with a stronger hypothesis than what is actually used in their proof. The following is really what is proved, and what we will use to prove (5.2).

(5.3) Lemma [BK, Lemma (3.3)]. Let $T$ be a bijection of a set $X$ with \# $N_{n}<$ $\infty$ for each $n$. Let $\varphi$ be a T-commuting involution of the set $X$, such that for each $k \geq 0$

$$
\zeta_{\varphi \circ T^{2^{k}}}=\zeta_{T^{2^{k}}}
$$

Then $\varphi$ satisfies the SGCC. (By an involution here we simply mean an involution as a map.)

Their proof of (5.3) is obtained by an analysis of the restrictions, that the zeta function hypothesis imposes, on the way that an involution can permute cycles. 
Now, any power, $\Sigma_{A^{n}}$, of a Bernoulli shift is again a Bernoulli shift. By virtue of this and (5.3), we need only show that for any Bernoulli shift $\Sigma_{A}$ and involution $\varphi$,

for each $w \in \mathrm{WPS}_{A}$.

$$
\zeta_{\varphi_{w} \circ\left(\sigma_{A_{0}}\right) w}=\zeta_{\left(\sigma_{A_{0}}\right) w}
$$

For this purpose, it suffices to find a Markov chain defined by some $B$ such that

$$
\zeta_{B}=\zeta_{A}
$$

and

$$
\zeta_{\left(\sigma_{B_{0}}\right)_{w}}=\zeta_{\varphi_{u}, \circ\left(\sigma_{A_{0}}\right)_{w}}
$$

for each $w \in \mathrm{WPS}_{A}$. (Here $A$ and $B$ are stochastic forms.) $\Sigma_{B}$ will really represent a Markov chain structure on " $\left(\Sigma_{A_{0}}, \varphi \circ \sigma_{A_{0}}, \mu_{A}\right)$."

We may assume that $A$ is a stochastic form and that (by [BLR, 2.9]), $\varphi$ is a 1-block map and therefore given by an involution of the set of edges of $G(A)$, although $A$ need not be $1 \times 1$ now. Since the stochastic form is unique, we have $\mathrm{wt}_{A}(\varphi(e))=\mathrm{wt}_{A}(e)$ for each edge $e$. We define $B$ as follows: $G(B)$ is obtained from $G(A)$ by replacing each edge $e$, from state $I$ to state $J$, by and edge, $f(e)$, from $I$ to $\underline{t}(\varphi(e))$ and setting $\mathrm{wt}_{B}(f(e))=\mathrm{wt}_{A}(e)$.

Note that $B^{2}=A^{2}$ by virtue of the bijections:

$$
E_{I J}\left(A^{2}\right) \rightarrow E_{I J}\left(B^{2}\right), \quad e_{1} e_{2} \mapsto f\left(e_{1}\right) f\left(\varphi\left(e_{2}\right)\right) .
$$

Thus, since $A$ is mixing, so is $B$ (all we really care about is that $B$ is irreducible).

Now, define $\bar{f}: \Sigma_{A_{0}} \rightarrow \Sigma_{B_{0}}$ by

$$
\left.\bar{f}(x)_{i}=f\left(\left(\varphi \circ \sigma_{A_{0}}\right)^{i}(x)\right)_{0}\right)= \begin{cases}f\left(x_{i}\right) & \text { if } i \text { is even } \\ f\left(\varphi x_{i}\right) & \text { if } i \text { is odd }\end{cases}
$$

Now, $\bar{f}$ satisfies

$$
\sigma_{B_{0}}=\bar{f} \circ\left(\varphi \circ \sigma_{A_{0}}\right) \circ \bar{f}^{-1},
$$

and thus is a conjugacy of bijections (in fact a topological conjugacy) between $\left(\Sigma_{B_{0}}, \sigma_{B_{0}}\right)$ and $\left(\Sigma_{A_{0}}, \varphi \circ \sigma_{A_{0}}\right)$. Also, since for each edge $e \in E(A)$

$$
\mathrm{wt}_{B}(f(e))=\mathrm{wt}_{A}(e)=\mathrm{wt}_{A}(\varphi(e)),
$$

$\bar{f}$ induces a conjugacy between $\left(\mathscr{C}_{w}(B),\left(\sigma_{B_{0}}\right)_{w}\right)$ and $\left(\mathscr{C}_{w}(A), \varphi_{w} \circ\left(\sigma_{A_{0}}\right)_{w}\right)$, and thus

$$
\zeta_{\left(\sigma B_{0}\right)_{w}}=\zeta_{\varphi_{w} \circ\left(\sigma_{A_{0}}\right)_{w}}
$$

So, we have established (12).

Now,

$$
\sigma_{B_{0}}^{2}=\bar{f} \circ\left(\varphi \circ \sigma_{A_{0}}\right)^{2} \circ \bar{f}^{-1}=\bar{f} \circ\left(\sigma_{A_{0}}\right)^{2} \circ \bar{f}^{-1} .
$$

Since $\bar{f}$ carries the Markov measure defined by $A$ to the Markov measure defined by $B$, we get that $\Sigma_{B^{2}}$ and $\Sigma_{A^{2}}$ are block isomorphic. (In fact, $B^{2}=A^{2}$ as shown above.) So, $B^{2}$ and $A^{2}$ have the same stochastic zeta function and thus

$$
\operatorname{det}\left(I-A_{t}^{2} s\right)=\operatorname{det}\left(I-B_{t}^{2} s\right), \quad \text { for all } s, t \in \mathbf{R} .
$$


Since the stochastic zeta function is invariant under block isomorphism and since $\Sigma_{A}$ is Bernoulli, we may assume that $A$ is $1 \times 1$, i.e., $A \in Z^{+}[\exp ]$. Thus, for each $t, B_{t}^{2}$ can have only one nonzero eigenvalue, namely $A_{t}^{2}$. So, $B_{t}$ can have only one nonzero eigenvalue for each $t: f(t) A_{t}, f(t) \in\{ \pm 1\}$. But since $B_{t}$ is a nonnegative matrix, we get by the Perron-Frobenius Theorem that $f(t) \equiv 1$. So, $\zeta_{B}=\zeta_{A}$ i.e., (11). So, we have established (11) and (12), and this completes the proof of $(5.2)$.

Finally, we remark that it is not sufficient to replace the SGCC condition at each wps with SGCC conditions at each face. For example, in the Bernoulli shift defined by

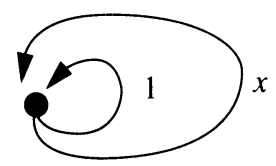

the permutation that fixes the cycles of lengths 1 and 2 and shifts forward one cycle of length 3 and shifts backward the other cycle of length 3 , satisfies the SGCC on each face, but not on each wps.

\section{APPENDIX A}

The induced SE is well defined.

Proposition A [Wa2]. If $(R, S)$ and $\left(R^{\prime}, S^{\prime}\right)$ are $S E$ 's induced by the same $\varphi$ with the same lag, then for some monomial $u$

$$
R^{\prime}=u R, \quad S^{\prime}=u^{-1} S .
$$

Proof. Suppose that $(R, S)$ and $\left(R^{\prime}, S^{\prime}\right)$ are SE's induced by the same $\varphi$ with the same lag $L$; we may as well assume that $L=1$; if not, replace $A$ by $A^{L}$. Then for some choice of identifications, $\varphi=c(R, S)=c\left(R^{\prime}, S^{\prime}\right)$. Let $(r(e), s(e))$ implement the identifications for $(R, S)$ and let $\left(r^{\prime}(e), s^{\prime}(e)\right)$ implement the identifications for $\left(R^{\prime}, S^{\prime}\right)$.

Fix an edge $e \in E(A)$ and state $J \in S(A)$. Let $I=\underline{t}(e)$. Define

$$
F_{e, J}=\left\{f \in E(A): \underline{t}(f)=J \text { and }(\varphi(x))_{0}=f \text { for some } x \text { with } x_{0}=e\right\} .
$$

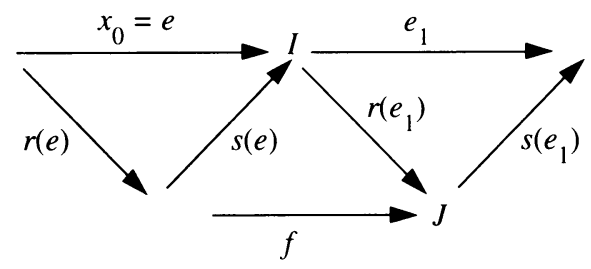

Then

$$
\sum_{f \in F_{e}, J} \mathrm{wt}(f)=\mathrm{wt}_{S}(s(e)) R_{I J}
$$

But since $F_{e, J}$ depends only on $\varphi$ and not on the specific SE induced by $\varphi$, we also get

$$
\sum_{f \in F_{e, J}} \operatorname{wt}(f)=\mathrm{wt}_{S^{\prime}}\left(s^{\prime}(e)\right) R_{I J}^{\prime} .
$$

So, $\mathrm{wt}_{S}(s(e)) R_{I J}=\mathrm{wt}_{S^{\prime}}\left(s^{\prime}(e)\right) R_{I J}^{\prime}$. 
Thus $R_{I J}=0$ iff $R_{I J}^{\prime}=0$ and if $R_{I J} \neq 0$, then

$$
\frac{R_{I J}^{\prime}}{R_{I J}}=\frac{\mathrm{wt}_{S}(s(e))}{\mathrm{wt}_{S^{\prime}}\left(s^{\prime}(e)\right)} .
$$

Since the right-hand side of this equation is independent of $J$, for some monomial $u_{I}$

$$
R_{I J}^{\prime} / R_{I J}=u_{I}
$$

Since this is true for all states $I$ we get $R^{\prime}=U R$ where $U$ is a diagonal matrix with monomial entries (namely, $U_{I I}=u_{I}$ ).

Likewise, $S^{\prime}=S V$ for some diagonal matrix $V$ with monomial entries.

It remains to show that $U=V^{-1}$ and $U$ is a scalar matrix (i.e., some monomial times the identity). We prove this as follows.

Let $I, J, K \in S(A)$. Then since $(\varphi)^{-1} \in c(S, R)$,

$$
S_{I J} R_{J K}=\sum \mathrm{wt}(e)
$$

where the sum ranges over all edges $e \in E_{I K}$ such that for some $x \in \Sigma_{A}$ with $x_{0}=e, \underline{i}\left(\left(\varphi^{-1}(x)\right)_{0}\right)=J$.

Since the right-hand side of this equation depends only on $\varphi$, and not on the specific induced SE, we get

$$
S_{I J} R_{J K}=S_{I J}^{\prime} R_{J K}^{\prime}=S_{I J} V_{J J} U_{J J} R_{J K}
$$

Thus $V_{J J} U_{J J}=1$ whenever $S_{I J} R_{J K} \neq 0$ for some $I, K$. Since $A=R S$ is irreducible, no row of $R$ or column of $S$ can be 0 . Thus $U=V^{-1}$.

Also, for states $I, J, K \in S(A)$, since $\varphi \in c(R, S)$,

$$
R_{I J} S_{J K}=\sum \mathrm{wt}(e)
$$

where the sum ranges over all edges $e \in E_{I K}$ such that for some $x \in \Sigma_{A}$ with $x_{0}=e, \underline{i}\left((\varphi(x))_{0}\right)=J$. Thus

$$
R_{I J} S_{J K}=R_{I J}^{\prime} S_{J K}^{\prime}=U_{I I} R_{I J} S_{J K} V_{K K}=U_{I I} R_{I J} S_{J K}\left(U_{K K}\right)^{-1} .
$$

Whenever $A_{I K} \neq 0$, we get that $R_{I J} S_{J K} \neq 0$ for some $J$ and thus $U_{I I}=U_{K K}$. Since $A$ is irreducible, all of the diagonal entries of $U$ coincide i.e., $U$ is a scalar matrix, as desired.

\section{APPENDIX B. Frame CODES ARE INERT}

We assume that the reader is familiar with higher order SFT's and higher order Markov measures. Recall that simple automorphism are inert.

Proposition B. Every frame code is simple i.e., block isomorphic to an automorphism $c(I, \bar{A})$ for some $A$.

Proof. We modify the proof of the analogous result in the topological setting [B, Appendix]. Recall the formulation of the frame code $\varphi$ described in the proof of (4.1). Introduce new (i.e., different from the edges of $G(A)$ ) symbols $b_{1} \cdots b_{l}, \bar{b}_{l}$ where $l=l(\gamma)=l(\eta)$. For each $x \in \Sigma_{A}$ define $\pi_{1}(x)$ by replacing $\gamma$ by $b_{1} \cdots b_{l-1} b_{l}$ whenever $\gamma$ is the central block of 5 copies of $\gamma$ and $\eta$, and replacing $\eta$ by $b_{1} \cdots b_{l-1} \bar{b}_{l}$ whenever $\eta$ is the central block of 5 copies of $\gamma$ and $\eta$. The map $\pi_{1}$ is well-defined, commutes with the shift and is 
1-1. The $\pi_{1}$-image of $\Sigma_{A}$ is a higher order SFT $\Lambda$ and the map $\pi_{1}$ carries the Markov measure on $\Sigma_{A}$ to a higher order Markov measure $\mu$ on $\Lambda$. Let $n$ denote the order of this Markov measure. Now, construct a finite directed graph as follows: define an equivalence relation on the blocks of length $n$ which appear in elements of $\Lambda$; say that $u$ is equivalent to $v$ if the conditional probabilities $p(a \mid u)=p(a \mid v)$ for all symbols $a$ (the conditional probabilities are those determined by $\mu$ ). The states of the graph $G$ are the equivalence classes of these blocks; and for each equivalence class $[u], u=u_{1} \cdots u_{n}$ and each symbol $a$ such that $p(a \mid u)>0$, define an edge $e$ from $[u]$ to $\left[u_{2} \cdots u_{n} a\right]$ and define the weight of this edge to be $p(a \mid u)$. We write such an edge $e=$ $e([u], a)$. This defines a (stochastic) $\mathrm{Z}^{+}[\exp ]-$ matrix $B$ and there is a measurepreserving shift-commuting map $\pi_{2}: \Sigma_{B} \rightarrow \Lambda$ defined by mapping each edge $e=e([u], a)$ to the symbol $a$. Now it is straightforward to check that $\pi_{2}$ is $1-1$ and that $\pi \equiv\left(\pi_{2}\right)^{-1} \circ \pi_{1}: \Sigma_{A} \rightarrow \Sigma_{B}$ is a block isomorphism. And $\pi \circ \varphi \circ(\pi)^{-1}$ is the automorphism which switches $e\left([u], b_{l}\right)$ and $e\left([u], \bar{b}_{l}\right)$ whenever $p\left(b_{l} \mid u\right)>$ 0 . Since $\left[u_{2} \cdots u_{n} b_{l}\right]=\left[u_{2} \cdots u_{n} \bar{b}_{l}\right]$, this map belongs to $c(I, \bar{B})$. So the frame code $\varphi$ is simple.

\section{REFERENCES}

[AM] R. Adler and B. Marcus, Topological entropy and equivalence of dynamical systems, Mem. Amer. Math. Soc. No. 219 (1979).

[B] M. Boyle, Nasu's simple automorphisms, Proc. Maryland Special Year in Dynamical Systems 1986-1987, Lecture Notes in Math., vol. 1342, Springer-Verlag, 1988, pp. 22-32.

[BMT] M. Boyle, B. Marcus and P. Trow, Resolving maps and the dimension group for shifts of finite type, Mem. Amer. Math. Soc. No. 377 (1987).

[BK] M. Boyle and W. Krieger, Periodic points and automorphisms of the shift, Trans. Amer. Math. Soc. 302 (1987), 125-149.

[BL] R. Bowen and O. Lanford, Zeta functions of restrictions of the shift transformation, Proc. Sympos. Pure Math., vol. 14, Amer. Math. Soc., Providence, R. I., 1970, pp. 43-50.

[BLR] M. Boyle, D. Lind and D. Rudolph, The automorphism group of a shift of finite type, Trans. Amer. Math. Soc. 306 (1988), 71-114.

[JKKMS] A. del Junco, M. Keane, B. Kitchens, B. Marcus and L. Swanson, Continuous homomorphisms of Bernoulli schemes, Progress in Math. 10, Birkhäuser, Boston, Mass., 1981, pp. 91-111.

[KR] H. Kim and F. Roush, On the structure of inert automorphisms of subshifts, Pure Math. Appl. 2 (1991), 3-22.

[KRW] H. Kim, F. Roush, and J. Wagoner, Automorphisms of the dimension group and gyration numbers, J. Amer. Math. Soc. 5 (1992), 191-212.

[Ki] B. Kitchens, Linear algebra and subshifis of finite type, Proc. Conf. Modern Analysis and Probability, Contemp. Math., vol. 26, Amer. Math. Soc., Providence, R. I., 1984, pp. 231248.

[Kr] W. Krieger, On the finitary isomorphisms of Markov shifts that have finite expected coding time, Z. Wahrsch. Verw Gebiete 65 (1983), 323-328.

[MT1] B. Marcus and S. Tuncel, The weight-per-symbol polytope and scaffolds of invariants associated with Markov chains, Ergodic Theory Dynamical Systems 11 (1991), 129-180.

[MT2] B. Marcus and S. Tuncel, Entropy at a weight-per-symbol and embeddings of Markov chains, Invent. Math. 102 (1990), 235-266.

[N] M. Nasu, Topological conjugacy for sofic systems and extensions of automorphisms of finite subsystems of topological Markov shifts, Proc. Maryland Special Year in Dynamical Systems 1986-1987, Lecture Notes in Math., vol. 1342, Springer-Verlag, 1988. 
[PS] W. Parry and K. Schmidt, Natural coefficients and invariants for Markov shifts, Invent. Math. 76 (1984), 15-32.

[PT1] W. Parry and S. Tuncel, On the classification of Markov chains by finite equivalence, Ergodic Theory Dynamical Systems 1 (1981), 303-335.

[PT2] - On the stochastic and topological structure of Markov chains, Bull. London Math. Soc. 14 (1982), 16-27.

[PW] W. Parry and R. Williams, Block coding and a zeta function for finite Markov chains, Proc. London Math. Soc. (3) 35 (1977), 483-495.

[Se] E. Seneta, Non-negative matrices and Markov chains, Springer, New York, 1981.

[T1] S. Tuncel, Conditional pressure and coding, Israel J. Math. 39 (1981), 101-112.

[T2] _ A dimension, dimension modules, and Markov chains, Proc. London Math. Soc. 46 (1983), 100-116.

[Wa1] J. Wagoner, Markov partitions and $K_{2}$, Publ. Math. Inst. Hautes Etudes Sci. 65 (1987), 91-129.

[Wa2] _ in preparation.

[Wi] R. Williams, Classification of shifts of finite type, Ann. of Math. 98 (1973), 120-153; Errata, Ann. of Math. 99 (1974), 380-381.

Institut fur Angewandte Mathematik, Universitat Heidelberg, Heidelberg D-6900, GERMANY

E-mail address: ae5@dhdurz1.bitnet

ibM Research Division, Almaden Research Center, 650 Harry Road, San Jose, CaliFORNIA 95120-6099

E-mail address: marcus@almaden.ibm.com

Department of Mathematics, University of Washington, Seattle, Washington 98195

E-mail address: tuncel\%math.wshington.edu@beaver.cs.wshington.edu 